\title{
On quantum quaternion spheres
}

\author{
Bipul SAURABH
}

July 19, 2021

\begin{abstract}
For the quantum symplectic group $S P_{q}(2 n)$, we describe the $C^{*}$-algebra of continuous functions on the quotient space $S P_{q}(2 n) / S P_{q}(2 n-2)$ as a universal $C^{*}$-algebra given by a finite set of generators and relations. The proof involves a careful analysis of the relations, and use of the branching rules for representations of the symplectic group due to Zhelobenko. We then exhibit a set of generators of the $K$-groups of this $C^{*}$-algebra in terms of generators of the $C^{*}$-algebra.
\end{abstract}

AMS Subject Classification No.: 58B34, 46L87, 19K33

Keywords. quantum group, symplectic group, universal $C^{*}$-algebra.

\section{Introduction}

The theory of quantum groups was first studied in the topological setting independently by Woronowicz [19] and Vaksman \& Soibelman [17]. Both of these papers studied the quantum $S U(2)$ group. Later Woronowicz developed the theory of compact quantum groups and their representation theory. The notions of quantum subgroups and quantum homogeneous spaces were soon introduced by Podleś ([15]). The most well-known example of compact quantum group is the $q$-deformation of the $S U(n)$ group whose representation theory was obtained by Vaksman \& Soibelman (see [18]). Later, Korogodski \& Soibelman ([11]) gave a complete classification of all the irreducible representations of the $C^{*}$-algebra $C\left(G_{q}\right)$ where $G_{q}$ is the $q$-deformation of a classical simply connected semisimple compact Lie group. Vaksman \& Soibelman [18] further studied quantum homogeneous space $S U_{q}(n) / S U_{q}(n-1)$ and obtained its irreducible representations. Quantum analogs of the Stiefel manifolds $S U_{q}(n) / S U_{q}(n-m)$ were introduced by Podkolzin \& Vainerman [14] who classified the irreducible representations of the $C^{*}$-algebras underlying such manifolds.

Given a compact quantum group $G$ and a subgroup $H$, the $C^{*}$-algebra $C(G / H)$ of the quotient space $G / H$ is defined to be a certain $C^{*}$-subalgebra of $C(G)$. Extending the ideas of Korogodski \& Soibelman in ([11]), Neshveyev \& Tuset ([12]) gave a complete classification of the irreducible representations of the $C^{*}$-algebra $C\left(G_{q} / H_{q}\right)$ where $G_{q}$ is the $q$-deformation of a 
simply connected semisimple compact Lie group and $H_{q}$ is the $q$-deformation of a closed PoissonLie subgroup $H$ of $G$. To understand the structure of these $C^{*}$-algebras further, the next thing to look for would be their $K$-groups. Vaksman \& Soibelman [18] determined $K$-groups of the stiefel manifolds $S U_{q}(n) / S U_{q}(n-1)$ which are quantum analogues of the classical odd dimensional spheres. Chakraborty \& Sundar [5] computed the $K$-theory of the Stiefel manifolds $S U_{q}(n) / S U_{q}(n-2)$ and as a consequence they found the $K$-theory of $S U_{q}(3)$. Then Neshveyev \& Tuset proved in the above mentioned paper ([12]) that $C\left(G_{q} / H_{q}\right)$ is $K K$-equivalent to the classical counterpart $C(G / H)$.

Here one question arises. Can we describe the algebra of functions on a quantum group $G_{q}$ or its quotient spaces $G_{q} / H_{q}$ in terms of a finite set of generators and relations? Given a semisimple compact Lie group $G$, the algebra of functions $C\left(G_{q}\right)$ on its $q$-deformation $G_{q}$ can be defined as the universal enveloping $C^{*}$-algebra generated by matrix co-efficients of all finite dimensional representations of Quantized universal enveloping algebra $U_{q}(g)$ where $g$ is the Lie algebra of $G$. Similarly, for a subgroup $H_{q}$ of $G_{q}$, the algebra of functions $C\left(G_{q} / H_{q}\right)$ on the quotient space $G_{q} / H_{q}$ can be described as the subalgebra of $C\left(G_{q}\right)$ generated by matrix elements of certain finite dimensional representations of Quantized universal enveloping algebra $U_{q}(g)$. But how to get a finite set of generators and relations to describe these $C^{*}$ - algebras is not clear. Korogodski and Soibelman described $C\left(S U_{q}(2)\right)$ in terms of finite set of generators and relations (see [11] theorem 3.0.1). For $C\left(S U_{q}(n)\right)$ case, it is proved by Woronowicz [20]. Another approach called FRT approach to define quantum deformation of a simple Lie group is due to Faddeev, Reshetikhin and Takhtajan. They started with a finite set of generators and relations based on quantum $R$-matrix of a simple Lie algebra to define the algebra of functions on the quantum group. Through a pairing between this algebra of functions and Quantized universal enveloping algebra, one can view this algebra of functions as certain subalgebra of dual Hopf-algebra of Quantized universal enveloping algebra. This relates FRT approach with the previous one due to V. Drinfeld. One difficulty with FRT approach is that there is no formula for the quantum $R$-matrix of an arbitrary simple Lie algebra. Also, even in this approach, the question of describing the algebra of functions on homogeneous spaces does not have an answer yet.

Many of the $C^{*}$-algebras arising in the context of quantum groups and their homogeneous spaces are universal $C^{*}$-algebras given by finite sets of generators and relations. This fact along with a knowledge of their generators and relations turn out to be extremely useful in studying these objects further as well as in doing various computations involving them. For example, the description of $C\left(S U_{q}(2)\right)$ in terms of its generators and relations was used by Vaksman \& Soibelman [18] to compute its K-theory. Later these generators and relations were used by various authors $([3], 6],[7,[13])$ to study the quantum group $S U_{q}(2)$ in the context of non commutative geometry. Similarly the description of the quantum odd dimensional spheres as universal $C^{*}$-algebras given by finite sets of generators and relations was used by Vaksman \& 
Soibelman [18 to compute its K-theory; subsequently by Hong \& Szymanski [9] to show that it can be realized as a graph $C^{*}$-algebra, which in turn gives a great deal of insight into these $C^{*}$-algebras, and finally by Chakraborty \& Pal [4] and by Pal \& Sundar [13] to study spectral triples for these spaces.

Describing a $C^{*}$-algebra in terms of generators and relations is a rather difficult and intractable problem in general. For $C^{*}$-algebra of 'continuous functions' on quantum groups or their homogeneous spaces, it is often the case that they are generated by certain matrix elements of representations. But there is no general results in this direction. In the present paper, we attempt this problem for the $C^{*}$-algebra corresponding to the quantum Stiefel manifold $S P_{q}(2 n) / S P_{q}(2 n-2)$. We first show that $C\left(S P_{q}(2 n) / S P_{q}(2 n-2)\right)$ is the $C^{*}$-subalgebra of $C\left(S P_{q}(2 n)\right)$ generated by elements of the first and last row of the fundamental co-representation of the quantum symplectic group $S P_{q}(2 n)$. We then get a finite set of relations satisfied by these elements. Then making use of the results of Neshveyev \& Tuset, we prove that $C\left(S P_{q}(2 n) / S P_{q}(2 n-2)\right)$ is the universal $C^{*}$-algebra given by a finite set of generators and relations. We then go on to exhibit generators of its $K$-groups in terms of the generators of this $C^{*}$-algebra.

Here is brief outline of how the paper is organized. In subsection 2.1, we start by recalling $q$ deformation of the symplectic group $S P_{q}(2 n)$ at the $C^{*}$-algebra level and describe its quantum group structure. In subsection (2.2) and (2.3), we first write down explicitly the pairing between the quantum universal enveloping algebra $U_{q}(s p(2 n))$ and the co-ordinate algebra $O\left(S P_{q}(2 n)\right)$, which helps us write down all elementary representations. An application of the result of Korogodski \& Soibelman then gives us a complete list of all irreducible representations of $C\left(S P_{q}(2 n)\right)$, which in turn provides us with a faithful representation on a Hilbert space. In subsection 2.4, extending the ideas of Chakraborty \& Pal [2], we associate certain diagrams with these irreducible representations.

In section 3 , we consider the quantum homogeneous space $S P_{q}(2 n) / S P_{q}(2 n-2)$ and show that the $C^{*}$-algebra underlying this manifolds is generated by a finite set of generators. Here we use branching rules for representations of the symplectic group due to Zhelobenko [21]. In section 4 , we define the $C^{*}$-algebra $C\left(H_{q}^{2 n}\right)$ of continuous functions on the quantum quaternion sphere as a universal $C^{*}$-algebra with $2 n$ generators satisfying a certain set of relations. We then study these relations and find all irreducible representations of the $C^{*}$-algebra $C\left(H_{q}^{2 n}\right)$. We then show that the $C^{*}$-algebra $C\left(S P_{q}(2 n) / S P_{q}(2 n-2)\right)$ is a homomorphic image of $C\left(H_{q}^{2 n}\right)$. This is accomplished by showing that the generators of $C\left(S P_{q}(2 n) / S P_{q}(2 n-2)\right)$ obtained in section 3 satisfy the defining relations of $C\left(H_{q}^{2 n}\right)$. Finally, by comparing the irreducible representations of the two $C^{*}$-algebras, we conclude that they are isomorphic. Here we use Neshveyev \& Tuset's results on irreducible representations of a quotient space. Finally, in section 5, we find a chain of short exact sequences for $C\left(S P_{q}(2 n) / S P_{q}(2 n-2)\right)$. Utilizing these, we compute their $K$-groups with explicit generators. Here we should remark that these 
$K$-groups are already known, thanks to the $K K$-equivalence of $C\left(S P_{q}(2 n) / S P_{q}(2 n-2)\right)$ and $C(S P(2 n) / S P(2 n-2))$ proved in [12]. But the advantage of our computation here is that we produce explicit generators of these $K$-groups which can come in handy in many situations.

A word about notations. It is unfortunate but very common to use the notation $s p(2 n, \mathbb{C})$ to describe the type $C_{n}$ groups at the Lie algebra level, while one switches to $S P(n)$ at the group level. Here we have used $2 n$ at both places, i.e. $U_{q}(s p(2 n))$ denotes the Quantized universal enveloping algebra of type $C_{n}$ and $S P_{q}(2 n)$ denotes the same quantum group at the function algebra level. Throughout this paper, $q$ will denote a real number in the interval $(0,1)$ and $C$ is used to denote a generic constant. The standard bases of the Hilbert spaces $\ell^{2}(\mathbb{N})$ and $\ell^{2}(\mathbb{Z})$ will be denoted by $\left\{e_{n}: n \in \mathbb{N}\right\}$ and $\left\{e_{n}: n \in \mathbb{Z}\right\}$ respectively.

\section{Quantum symplectic group}

\subsection{The $C^{*}$-algebra $C\left(S P_{q}(2 n)\right)$}

In this subsection, we briefly recall from [10] various algebras and maps related to the compact quantum group $S P_{q}(2 n)$. We set up some notation that will be used throughout this paper. Define,

$$
\begin{aligned}
i^{\prime} & =2 n+1-i \\
\rho_{i} & =n+1-i \quad \text { if } i \leq n . \\
\rho_{i^{\prime}} & =-\rho_{i} . \\
\epsilon_{i} & = \begin{cases}1 & \text { if } 1 \leq i \leq n, \\
-1 & \text { if } n+1 \leq i \leq 2 n, \\
C_{j}^{i} & =\epsilon_{i} \delta_{i j} q^{-\rho_{i}} . \\
\theta(i) & = \begin{cases}0 & \text { if } i \leq 0, \\
1 & \text { if } i>0,\end{cases} \\
R_{m n}^{i j} & =q^{\delta_{i j}-\delta_{i j^{\prime}}} \delta_{i m} \delta_{j n}+\left(q-q^{-1}\right) \theta(i-m)\left(\delta_{j m} \delta_{i n}+C_{j}^{i} C_{n}^{m}\right) .\end{cases}
\end{aligned}
$$

Let $\mathbb{C}\left\langle u_{j}^{i}\right\rangle$ denote the free algebra with generators $u_{j}^{i}, i, j=1,2, \cdots 2 n$ and let $J(R)$ be the two sided ideal of $\mathbb{C}\left\langle u_{j}^{i}\right\rangle$ generated by the following elements,

$$
I_{s t}^{i j}=\sum_{k, l=1}^{2 n} R_{k l}^{j i} u_{s}^{k} u_{t}^{l}-R_{s t}^{l k} u_{k}^{i} u_{l}^{j}, \quad i, j, s, t=1,2 \cdots 2 n .
$$

Let $A(R)$ denote the quotient algebra $\mathbb{C}\left\langle u_{j}^{i}\right\rangle / J(R)$. The $2 n \times 2 n$ matrices $\left(\left(u_{j}^{i}\right)\right)$ and $\left(\left(C_{j}^{i}\right)\right)$ are denoted by $U$ and $C$ respectively. Define $J=\left\langle U C U^{t} C^{-1}-I, C U^{t} C^{-1} U-I\right\rangle$, the two 
sided ideal generated by entries of matrices $U C U^{t} C^{-1}-I$ and $C U^{t} C^{-1} U-I$. Let $O\left(S P_{q}(2 n)\right)$ denote the quotient algebra $A(R) / J$.

The algebra $O\left(S P_{q}(2 n)\right)$ is a Hopf-*algebra with co-multiplication $\Delta$, co-unit $\epsilon$, antipode $S$ and involution $*$ given on the generating elements by,

$$
\Delta\left(u_{j}^{i}\right)=\sum_{k=1}^{N} u_{k}^{i} \otimes u_{j}^{k}, \quad \epsilon\left(u_{j}^{i}\right)=\delta_{i j}, \quad S\left(u_{j}^{i}\right)=\epsilon_{i} \epsilon_{j} q^{\rho_{i}-\rho_{j}} u_{i^{\prime}}^{j^{\prime}}, \quad\left(u_{j}^{i}\right)^{*}=\epsilon_{i} \epsilon_{j} q^{\rho_{i}-\rho_{j}} u_{j^{\prime}}^{i^{\prime}} .
$$

Note that $U^{*}=C U^{t} C^{-1}$. Hence we have,

$$
U U^{*}=U^{*} U=I
$$

Now to make $O\left(S P_{q}(2 n)\right)$, a normed-*algebra, we define,

$$
\|a\|=\sup \left\{\|\pi(a)\|: \pi \text { is a representation of } O\left(S P_{q}(2 n)\right)\right\} .
$$

By (2.2), we have, $\left\|u_{j}^{i}\right\| \leq 1$, hence for all $a \in O\left(S P_{q}(2 n)\right)$, $\|a\|<\infty$. We denote by $C\left(S P_{q}(2 n)\right)$ the completion of $O\left(S P_{q}(2 n)\right)$. The pair $\left(C\left(S P_{q}(2 n)\right), \Delta\right)$ is a compact quantum group called a $q$-deformation of the symplectic group $S P_{q}(2 n)$. For more detail, see ([10], page 317-320, page 326).

\subsection{Pairing between $U_{q}\left(s p_{2 n}\right)$ and $\boldsymbol{O}\left(S P_{q}(2 n)\right)$}

Let $\left(\left(a_{i j}\right)\right)$ be the Cartan matrix of Lie algebra $s p(2 n)$ given by,

$$
a_{i j}= \begin{cases}2 & \text { if } i=j \\ -1 & \text { if } i=j+1 \\ -1 & \text { if } i=j-1, i \neq n-1 \\ -2 & \text { if } i=j-1=n-1 \\ 0 & \text { otherwise }\end{cases}
$$

Define $q_{i}=q^{d_{i}}$, where $d_{i}=1$ for $i=1,2, . . n-1$ and $d_{n}=2$. The quantized universal enveloping algebra (QUEA) $U_{q}\left(s p_{2 n}\right)$ is the universal algebra generated by $E_{i}, F_{i}, K_{i}$ and $K_{i}^{-1}, i=1, \ldots, \ell$, satisfying the following relations

$$
\begin{gathered}
K_{i} K_{j}=K_{j} K_{i}, \quad K_{i} K_{i}^{-1}=K_{i}^{-1} K_{i}=1, \\
K_{i} E_{j} K_{i}^{-1}=q_{i}^{a_{i j}} E_{j}, \quad K_{i} F_{j} K_{i}^{-1}=q_{i}^{a_{i j}} F_{j}, \\
E_{i} F_{j}-F_{j} E_{i}=\delta_{i j} \frac{K_{i}-K_{i}^{-1}}{q_{i}-q_{i}^{-1}}, \\
\sum_{r=0}^{1-a_{i j}}(-1)^{r}\left(\begin{array}{c}
1-a_{i j} \\
r
\end{array}\right)_{q_{i}} E_{i}^{1-a_{i j}-r} E_{j} E_{i}^{r}=0 \quad \forall i \neq j,
\end{gathered}
$$




$$
\sum_{r=0}^{1-a_{i j}}(-1)^{r}\left(\begin{array}{c}
1-a_{i j} \\
r
\end{array}\right)_{q_{i}} F_{i}^{1-a_{i j}-r} F_{j} F_{i}^{r}=0 \quad \forall i \neq j,
$$

where $\left(\begin{array}{l}n \\ r\end{array}\right)_{q}$ denote the $q$-binomial coefficients. Hopf *-structure comes from the following maps:

$$
\begin{gathered}
\Delta\left(K_{i}\right)=K_{i} \otimes K_{i}, \quad \Delta\left(K_{i}^{-1}\right)=K_{i}^{-1} \otimes K_{i}^{-1} \\
\Delta\left(E_{i}\right)=E_{i} \otimes K_{i}+1 \otimes E_{i}, \quad \Delta\left(F_{i}\right)=F_{i} \otimes 1+K_{i}^{-1} \otimes F_{i}, \\
\epsilon\left(K_{i}\right)=1, \quad \epsilon\left(E_{i}\right)=0=\epsilon\left(F_{i}\right), \\
S\left(K_{i}\right)=K_{i}^{-1}, \quad S\left(E_{i}\right)=-E_{i} K_{i}^{-1}, \quad S\left(F_{i}\right)=-K_{i} F_{i}, \\
K_{i}^{*}=K_{i}, \quad E_{i}^{*}=K_{i} F_{i}, \quad F_{i}^{*}=E_{i} K_{i}^{-1} .
\end{gathered}
$$

We refer the reader to [10] for a proof of the following theorem that gives a dual pairing between $U_{q}\left(s p_{2 n}\right)$ and $O\left(S P_{q}(2 n)\right)$.

Theorem 2.1. ([10]) There exist unique dual pairing $\langle\cdot, \cdot\rangle$ between the Hopf algebras and $U_{q}\left(s l_{2}\right)$ and $\mathrm{O}\left(S L_{q}(2)\right)$ and between $U_{q}\left(s p_{2 n}\right)$ and $\mathrm{O}\left(S P_{q}(2 n)\right)$ such that

$$
\left\langle f, u_{l}^{k}\right\rangle=t_{k l}(f) \quad \text { for } k, l=1,2, \ldots 2 n .
$$

where $t_{k l}$ is the matrix element of $T_{1}$, the vector representation of $U_{q}\left(s l_{2}\right)$ in first case and that of $U_{q}\left(s p_{2 n}\right)$ in second case.

We will explicitly describe $T_{1}$ for both cases and determine the pairing. For that, let $E_{i j}$ be the $2 n \times 2 n$ matrix with 1 in the $(i, j)^{t h}$ position and 0 elsewhere and $D_{j}$ be the diagonal matrix with $q$ in the $(j, j)^{t h}$ position and 1 elsewhere on the diagonal.

For the QUEA $U_{q}\left(s p_{2 n}\right)$, one has

$$
\left.\begin{array}{l}
T_{1}\left(K_{i}\right)=D_{i}^{-1} D_{i+1} D_{2 n-i}^{-1} D_{2 n-i+1} . \\
T_{1}\left(E_{i}\right)=E_{i+1, i}-E_{2 n-i+1,2 n-i} . \\
T_{1}\left(F_{i}\right)=E_{i, i+1}-E_{2 n-i, 2 n-i+1} .
\end{array}\right\} \text { for } \quad i \in\{1,2, \cdots, n-1\}
$$

and

$$
\left.T_{1}\left(K_{n}\right)=D_{n}^{-2} D_{n+1}^{2}, \quad T_{1}\left(E_{n}\right)=E_{n+1, n}, \quad T_{1}\left(F_{n}\right)=E_{n, n+1}\right\} \quad \text { for } \quad i=n .
$$

For $U_{q}\left(s l_{2}\right)$, one has (here $E_{i j}$ and $D_{j}$ are the $2 \times 2$ matrices.)

$$
T_{1}(K)=D_{1}^{-1} D_{2}, \quad T_{1}(E)=E_{2,1}, \quad T_{1}(F)=E_{1,2}
$$

For more detail, we refer to ([10], page 267-268). We will use these two pairing to write down irreducible representations of $O\left(S P_{q}(2 n)\right)$ which can be extended to $C\left(S P_{q}(2 n)\right)$ to get elementary representations of $C\left(S P_{q}(2 n)\right)$. 
Elementary representation of $C\left(S P_{q}(2 n)\right)$ : For $i \in\{1,2, \cdots, n\}$, let $\varphi_{i}: U_{q_{i}}\left(s l_{2}\right) \longrightarrow$ $U_{q}(s p(2 n))$ be a $*$-homomorphism given on generators of $U_{q_{i}}\left(s l_{2}\right)$ by,

$$
K \longmapsto K_{i}, \quad E \longmapsto E_{i}, \quad F \longmapsto F_{i}
$$

Consider the dual epimorphism,

$$
\varphi_{i}^{*}: C\left(S P_{q}(2 n)\right) \longrightarrow C\left(S U_{q_{i}}(2)\right)
$$

such that

$$
\left\langle f, \varphi_{i}^{*}\left(u_{n}^{m}\right)\right\rangle=\left\langle\varphi_{i}(f), u_{n}^{m}\right\rangle
$$

In particular,

$$
\left\langle K, \varphi_{i}^{*}\left(u_{n}^{m}\right)\right\rangle=\left\langle K_{i}, u_{n}^{m}\right\rangle, \quad\left\langle E, \varphi_{i}^{*}\left(u_{n}^{m}\right)\right\rangle=\left\langle E_{i}, u_{n}^{m}\right\rangle, \quad\left\langle F, \varphi_{i}^{*}\left(u_{n}^{m}\right)\right\rangle=\left\langle F_{i}, u_{n}^{m}\right\rangle .
$$

Remark 2.2. Initially $\varphi_{i}$ will induce an $*$ epimorphism from the Hopf- $*$ algebra $O\left(S P_{q}(2 n)\right)$ to $O\left(s l_{q_{i}}(2)\right)$ which when extended to $C\left(S P_{q}(2 n)\right)$ gives the above homomorphism at $C^{*}$-algebra level. For more detail see ([10], page 327) and [11].

Let $N$ be the number operator given by $N: e_{n} \mapsto n e_{n}$ and $S$ be the shift operator given by $S: e_{n} \mapsto e_{n-1}$ on $L_{2}(\mathbb{N})$. Denote by $\pi$ the following representation of $C\left(S U_{q}(2)\right)$ on $L_{2}(\mathbb{N})$;

$$
\pi\left(u_{l}^{k}\right)= \begin{cases}\sqrt{1-q^{2 N+2}} S & \text { if } k=l=1, \\ S^{*} \sqrt{1-q^{2 N+2}} & \text { if } k=l=2, \\ -q^{N+1} & \text { if } k=1, l=2, \\ q^{N} & \text { if } k=2, l=1, \\ \delta_{k l} & \text { otherwise . }\end{cases}
$$

Define, $\pi_{s_{i}}=\pi \circ \varphi_{i}^{*}$. Applying (2.3), we have, for $i=1,2, \cdots, n-1$,

$$
\pi_{s_{i}}\left(u_{l}^{k}\right)= \begin{cases}\sqrt{1-q^{2 N+2}} S & \text { if }(k, l)=(i, i) \text { or }(2 n-i, 2 n-i), \\ S^{*} \sqrt{1-q^{2 N+2}} & \text { if }(k, l)=(i+1, i+1) \text { or }(2 n-i+1,2 n-i+1), \\ -q^{N+1} & \text { if }(k, l)=(i, i+1), \\ q^{N} & \text { if }(k, l)=(i+1, i), \\ q^{N+1} & \text { if }(k, l)=(2 n-i, 2 n-i+1), \\ -q^{N} & \text { if }(k, l)=(2 n-i+1,2 n-i), \\ \delta_{k l} & \text { otherwise } .\end{cases}
$$


For $i=n$,

$$
\pi_{s_{n}}\left(u_{l}^{k}\right)= \begin{cases}\sqrt{1-q^{4 N+4}} S & \text { if }(k, l)=(n, n), \\ S^{*} \sqrt{1-q^{4 N+4}} & \text { if }(k, l)=(n+1, n+1), \\ -q^{2 N+2} & \text { if }(k, l)=(n, n+1), \\ q^{2 N} & \text { if }(k, l)=(n+1, n), \\ \delta_{k l} & \text { otherwise } .\end{cases}
$$

Each $\pi_{s_{i}}$ is an irreducible representation and is called an elementary representation of $C\left(S P_{q}(2 n)\right)$. For any two representations $\varphi$ and $\psi$ of $C\left(S P_{q}(2 n)\right)$ define, $\varphi * \psi:=(\varphi \otimes \psi) \circ \Delta$. Let $\mathrm{W}$ be the Weyl group of $s p_{2 n}$ and $\vartheta \in W$ such that $s_{i_{1}} s_{i_{2}} \ldots s_{i_{k}}$ is a reduced expression for $\vartheta$. Then $\pi_{\vartheta}=\pi_{s_{i_{1}}} * \pi_{s_{i_{2}}} * \cdots * \pi_{s_{i_{k}}}$ is an irreducible representation which is independent of the reduced expression. Now for $t=\left(t_{1}, t_{2}, \cdots, t_{n}\right) \in \mathbb{T}^{n}$, define the map $\tau_{t}: C\left(S P_{q}(2 n) \longrightarrow \mathbb{C}\right.$ by

$$
\tau_{t}\left(u_{j}^{i}\right)= \begin{cases}\overline{t_{i}} \delta_{i j} & \text { if } i \leq n \\ t_{2 n+1-i} \delta_{i j} & \text { if } i>n\end{cases}
$$

Then $\tau_{t}$ is a $*$-algebra homomorphism. For $t \in \mathbb{T}^{n}, \vartheta \in W$, let $\pi_{t, \vartheta}=\tau_{t} * \pi_{\vartheta}$.

We refer to ([1], page 121) for the following theorem.

Theorem 2.3. $\left\{\pi_{t, \vartheta} ; t \in \mathbb{T}^{n}, \vartheta \in W\right\}$ is a complete set of mutually inequivalent representations of $C\left(S P_{q}(2 n)\right)$.

\subsection{Representations of $C\left(S P_{q}(2 n)\right)$}

To write down all irreducible representations of $C\left(S P_{q}(2 n)\right)$, we need to recall a few facts on the Weyl group of $s p_{2 n}$ which we summarize below. Weyl group $W_{n}$ of $s p_{2 n}$ is a Coxeter group generated by $s_{1}, s_{2}, \ldots s_{n}$ satisfying the following relations:

$$
\begin{aligned}
s_{i}^{2} & =1 & & \text { for } i=1,2, \ldots n \\
s_{i} s_{i+1} s_{i} & =s_{i+1} s_{i} s_{i+1} & & \text { for } i=1,2, \ldots n-1 \\
s_{n-1} s_{n} s_{n-1} s_{n} & =s_{n} s_{n-1} s_{n} s_{n-1} & &
\end{aligned}
$$

The group $W_{n}$ can be embedded faithfully in $M_{n}(\mathbb{R})$ as,

$$
\begin{array}{ll}
s_{i}=I-E_{i, i}-E_{i+1, i+1}+E_{i, i+1}+E_{i+1, i}, & \text { for } \quad i=1,2, \ldots n-1, \\
s_{n}=I-2 E_{n, n}, & \text { for } \quad i=n .
\end{array}
$$

So, $W_{n}$ is isomorphic to a subgroup of $G L(n, \mathbb{R})$ generated by $s_{1}, s_{2}, \ldots s_{n}$. We refer to [8] for a proof of the following proposition. 
Proposition 2.4. $\quad$ 1. Let $\mathfrak{S}_{n}$ be the permutation group and $H_{n}$ be the $n$-fold direct product of the group $\{-1,1\} . \mathfrak{S}_{n}$ acts on $H_{n}$ by permuting its co-ordinates. Then $W_{n}=H_{n} \rtimes \mathfrak{S}_{n}$. In other word, $W_{n}$ is the set of $n \times n$ matrices having one non-zero entry in each row and each column which is either 1 or -1 .

2. Any element of $W_{n}$ can be written in the form: $\prod_{r=1}^{n} \psi_{r, k_{r}}^{\left(\epsilon_{r}\right)}$ where $\epsilon_{r} \in\{0,1,2\}$ and $r \leq k_{r} \leq n$ with the convention that,

$$
\psi_{r, k_{r}}^{\epsilon}= \begin{cases}s_{k_{r}-1} s_{k_{r}-2} \ldots s_{r} & \text { if } \epsilon=1, \\ s_{k_{r}} s_{k_{r}+1} \cdots \ldots s_{n-1} s_{n} s_{n-1} \cdots s_{k_{r}} s_{k_{r}-1} \cdots s_{r} & \text { if } \epsilon=2, \\ \text { empty string } & \text { if } \epsilon=0,\end{cases}
$$

Also, the above expression is a reduced expression.

3. The longest word of $W_{n}$ is $-I$ which can be written as $\prod_{r=1}^{n} \psi_{r, r}^{(2)}$. Also, $\left\{\psi_{r, r}^{(2)}\right\}_{i=1}^{n}$ commutes, hence $-I$ can be written as $\prod_{r=1}^{n} \psi_{n+1-r, n+1-r}^{(2)}$, which is a reduced expression.

Denote by $\mathscr{T}$ the Toeplitz algebra. Let $\vartheta$ be a word on $s_{1}, s_{2}, \ldots s_{n}$ of length $\ell(\vartheta)$. Then the map $\mathbb{T}^{n} \ni t \longmapsto \pi_{t, \vartheta}\left(u_{j}^{i}\right) \in \mathscr{T}^{\otimes \ell(\vartheta)}$ is continuous. Hence we have a homomorphism $\chi_{\vartheta}$ : $C\left(S P_{q}(2 n)\right) \longrightarrow C\left(\mathbb{T}^{n}\right) \otimes \mathscr{T}^{\otimes \ell(\vartheta)}$ such that $\chi_{\vartheta}(a)(t)=\pi_{t, \vartheta}(a)$, for all $a \in C\left(S P_{q}(2 n)\right)$.

Proposition 2.5. If $\vartheta^{\prime}$ is a subword of $\vartheta$ then $\chi_{\vartheta}$, and $\pi_{t, \vartheta}$, factor through $\chi_{\vartheta}$.

The proof of above proposition is straightforward, so we omit it.

Theorem 2.6. Let $\vartheta_{n}$ be the longest word of the Weyl group of $s p_{2 n}$ i.e

$$
\vartheta_{n}=\left(s_{n}\right)\left(s_{n-1} s_{n} s_{n-1}\right) \ldots\left(s_{2} \ldots s_{n} . . s_{2}\right)\left(s_{1} s_{2} \ldots s_{n-1} s_{n} s_{n-1} \ldots s_{1}\right) .
$$

Then the homomorphism

$$
\chi_{\vartheta_{n}}: C\left(S P_{q}(2 n)\right) \longrightarrow C\left(\mathbb{T}^{n}\right) \otimes \mathscr{T}^{\otimes \ell}\left(\vartheta_{n}\right)
$$

is faithful.

Proof: Any irreducible representation of $C\left(S P_{q}(2 n)\right)$ is of the form $\pi_{t, \vartheta}$ where $\vartheta$ is a word on $s_{1}, s_{2}, \ldots s_{n}$ and $t \in \mathbb{T}^{n}$. From proposition 2.4, it is clear that $\vartheta$ is a subword of $\vartheta_{n}$, hence $\pi_{t, \vartheta}$ factors through $\chi_{\vartheta_{n}}$ which shows that $\chi_{\vartheta_{n}}$ is faithful.

\subsection{Diagram representation}

At this point it will be useful to associate some diagrams with the above representations. We will use the scheme followed by Chakraborty \& Pal [2] with a few additions. For convenience, 
we use labeled arrows to represent operators as given in the following table.

\begin{tabular}{|c|c||c|c||c|c|}
\hline Arrow type & Operator & Arrow type & Operator & Arrow type & Operator \\
\hline$\longrightarrow$ & $I$ & & & & \\
$\stackrel{+}{\longrightarrow}$ & $S^{*} \sqrt{I-q^{2 N+2}}$ & $/+$ & $-q^{N+1}$ & $q^{N}$ \\
\hline- & $\sqrt{I-q^{2 N+2}} S$ & $/-$ & $q^{N+1}$ & $\searrow$ & $-q^{N}$ \\
\hline++ & $S^{*} \sqrt{I-q^{4 N+4}}$ & $/++$ & $-q^{2 N+2}$ & $\searrow+$ & $q^{2 N}$ \\
\hline-- & $\sqrt{I-q^{4 N+4}} S$ & & & & \\
\hline
\end{tabular}

Let us describe how to use a diagram to represent the irreducible $\pi_{s_{i}}$.

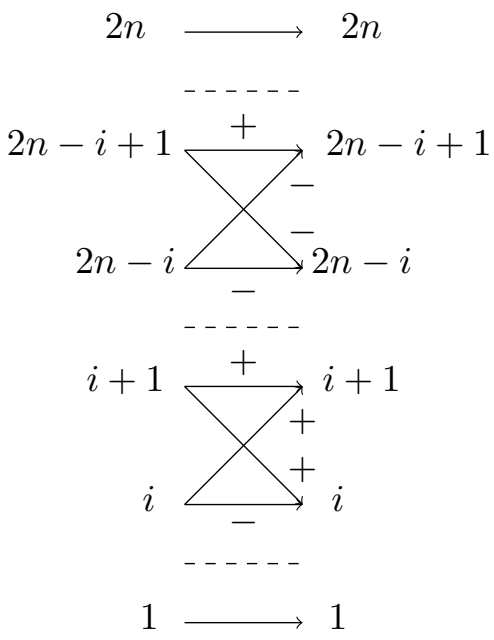

Diagram 1: $\psi_{s_{i}}, i \neq n$

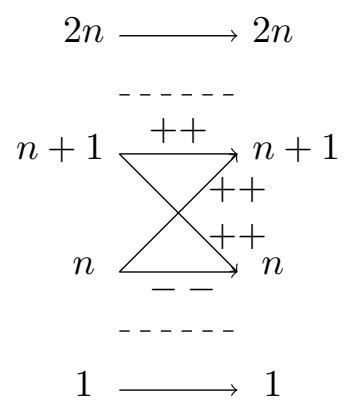

In these two diagrams, each path from a node $k$ on the left to a node $l$ on the right stands for an operator on $\mathcal{H}=\ell^{2}(\mathbb{N})$ given as in the table. Now $\pi_{s_{i}}\left(u_{l}^{k}\right)$ is the operator represented by the path from $k$ to $l$, and is zero if there is no such path. Thus, for example, $\pi_{s_{i}}\left(u_{1}^{1}\right)$ is $I$; 
$\pi_{s_{i}}\left(u_{1}^{2}\right)$ is zero whereas $\pi_{s_{i}}\left(u_{i+1}^{i}\right)=-q^{N+1}$ if $i>1$.

Next, let us explain how to represent $\pi_{s_{i}} * \pi_{s_{j}}$ by a diagram. Simply keep the two diagrams representing $\pi_{s_{i}}$ and $\pi_{s_{j}}$ adjacent to each other. Identify, for each row, the node on the right side of the diagram for $\pi_{s_{i}}$ with the corresponding node on the left in the diagram for $\pi_{s_{j}}$. Now, $\pi_{s_{i}} * \pi_{s_{j}}\left(u_{l}^{k}\right)$ would be an operator on the Hilbert space $\ell^{2}(\mathbb{N}) \otimes \ell^{2}(\mathbb{N})$ determined by all the paths from the node $k$ on the left to the node $l$ on the right. It would be zero if there is no such path and if there are more than one paths, then it would be the sum of the operators given by each such path. In this way, we can draw diagrams for each irreducible representation of $C\left(S P_{q}(2 n)\right)$.

Next, we come to $\chi_{\vartheta}$. The underlying Hilbert space now is $\ell^{2}\left(\mathbb{Z}^{n}\right) \otimes \ell^{2}(\mathbb{N})^{\otimes \ell(\vartheta)}$. To avoid any ambiguity, we have explicitly mentioned above the diagram the space on which the operator between nodes acts. As earlier, $\chi_{\vartheta}\left(u_{l}^{k}\right)$ stands for the operator on $\ell^{2}\left(\mathbb{Z}^{n}\right) \otimes \ell^{2}(\mathbb{N})^{\otimes \ell(\vartheta)}$ represented by the path from $k$ on the left to $l$ on the right. Note that we view $C\left(\mathbb{T}^{n}\right)$ as a subalgebra of $\mathcal{L}\left(\ell^{2}\left(\mathbb{Z}^{n}\right)\right)$.

The following diagram is for the representations $\chi_{\vartheta_{3}}$ of $C\left(S P_{q}(6)\right)$ where $\vartheta_{3}=s_{3} s_{2} s_{3} s_{2} s_{1} s_{2} s_{3} s_{2} s_{1}$.

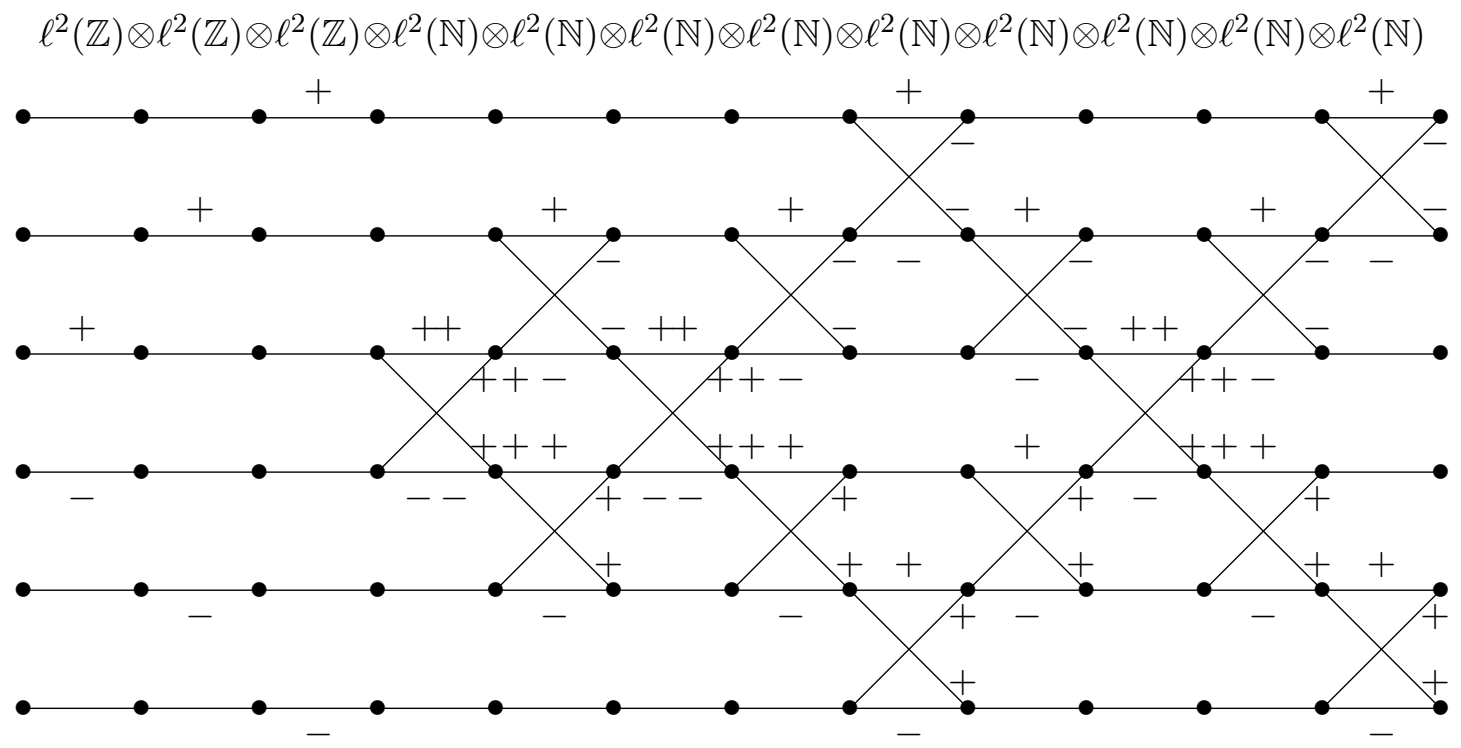

Diagram 3: $\chi_{\vartheta_{3}}$

Let $\omega_{n}=s_{1} s_{2} \cdots s_{n-1} s_{n} s_{n-1} \cdots s_{1}$. The following diagram is for the representation $\pi_{\omega_{3}}$ of $C\left(S P_{q}(6)\right)$. 


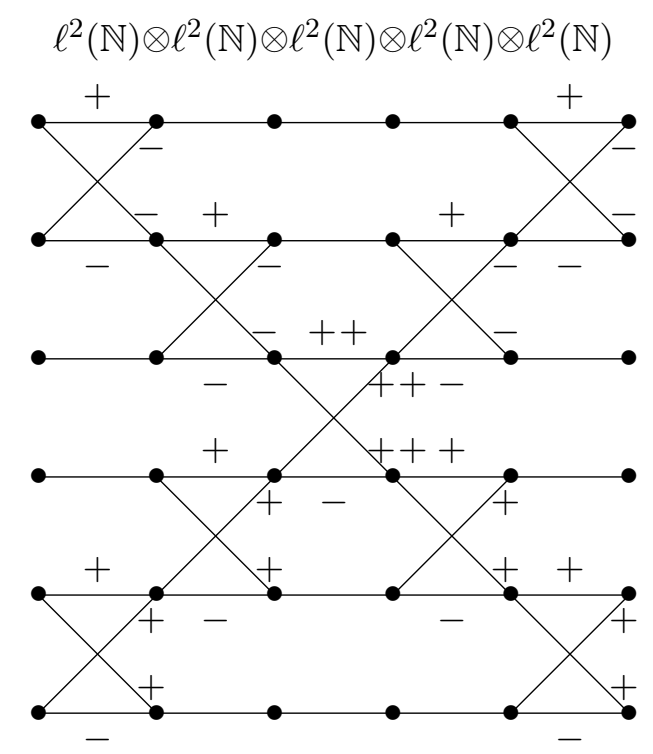

Diagram 4: $\pi_{\omega_{3}}$

\section{The quotient space $C\left(S P_{q}(2 n) / S P_{q}(2 n-2)\right)$}

In this section, we recall quantum homogeneous space $C\left(S P_{q}(2 n) / S P_{q}(2 n-2)\right)$ and show that it is the $C^{*}$ - subalgebra of $C\left(S P_{q}(2 n)\right)$ generated by $\left\{u_{m}^{1}, u_{m}^{2 n}: m \in\{1,2, \cdots 2 n\}\right\}$.

The Weyl group $W_{n-1}$ of $s p_{2 n-2}$ can be realized as a subgroup of the Weyl group $W_{n}$ of $s p_{2 n}$ generated by $s_{2}, s_{3}, \cdots s_{n}$ and hence, the longest word $\vartheta_{n-1}$ in $W_{n-1}$ is a subword of the longest word $\vartheta_{n}$ in $W_{n}$ which can easily be seen from proposition 2.4. This shows that $C\left(S P_{q}(2 n-2)\right)$ is a subgroup of $C\left(S P_{q}(2 n)\right)$, i.e. there is a $C^{*}$-epimorphism $\phi: C\left(S P_{q}(2 n)\right) \rightarrow C\left(S P_{q}(2 n-2)\right)$ obeying $\Delta \phi=(\phi \otimes \phi) \Delta$. More precisely, let $\sigma: \mathscr{T} \rightarrow \mathbb{C}$ is the homomorphism for which $\sigma(S)=$ 1. Define $\phi$ to be the restriction of $1^{\otimes n-1} \otimes e v_{1} \otimes 1^{\otimes(n-1)^{2}} \otimes \sigma^{\otimes(2 n-1)}$ to $\chi_{\vartheta_{n}}\left(C\left(S P_{q}(2 n)\right)\right)$ which is contained in $C\left(T^{n}\right) \otimes \mathscr{T}^{\otimes n^{2}}$. Here $e v_{1}$ denote the evaluation map at 1 i.e. $e v_{1}: C(T) \rightarrow \mathbb{C}$ such that $e v_{1}(f)=f(1)$. Image of $\phi$ is equal to $\chi_{\vartheta_{n-1}}\left(C\left(S P_{q}(2 n-2)\right)\right)$ as,

$$
\phi\left(\chi_{w_{n}}\left(u_{j}^{i}\right)\right)= \begin{cases}\chi_{\vartheta_{n-1}}\left(v_{j}^{i}\right), & \text { if } i \neq 1 \text { or } 2 n, \text { or } j \neq 1 \text { or } 2 n \\ \delta_{i j}, & \text { otherwise. }\end{cases}
$$

where $v_{j}^{i}$ are generators of $C\left(S P_{q}(2 n-2)\right)$. In such a case, one defines the quotient space $C\left(S P_{q}(2 n) / S P_{q}(2 n-2)\right)$ by,

$$
C\left(S P_{q}(2 n) / S P_{q}(2 n-2)\right)=\left\{a \in C\left(S P_{q}(2 n)\right):(\phi \otimes i d) \Delta(a)=I \otimes a\right\} .
$$

Theorem 3.1. The quotient space $C\left(S P_{q}(2 n) / S P_{q}(2 n-2)\right)$ is the $C^{*}$-algebra generated by $\left\{u_{m}^{1}, u_{m}^{2 n}: m \in\{1,2, \cdots 2 n\}\right\}$. 
We first prove one proposition which will be needed in the proof of above theorem. For that, define $\mathcal{B}$ to be the $*$-algebra generated by $\left\{u_{m}^{1}, u_{m}^{2 n}: m \in\{1,2, \cdots 2 n\}\right\}$. Consider $\mathcal{B}$ as a $U_{q}(s p(2 n))$-module with the following action:

$$
f(a)=(1 \otimes\langle f, .\rangle) \Delta a
$$

where $f \in U_{q}(\operatorname{sp}(2 n)), a \in \mathcal{B}$ and $\langle.,$.$\rangle is the pairing given in theorem 2.1. Fix a n$-tuple of integers of the form $(r, s, 0, \cdots, 0)$ satisfying $r \geq s$. We call $b \in \mathcal{B}$ highest weight vector with highest weight $(r, s, 0, \cdots, 0)$ if

$$
\begin{aligned}
K_{1}(b) & =q^{r-s} b, \\
K_{2}(b) & =q^{s} b, \\
K_{i}(b) & =b \quad \text { for all } \quad i \geq 2, \\
E_{i}(b) & =0 \quad \text { for all } \quad i \in\{1, \cdots, 2 n\} .
\end{aligned}
$$

Proposition 3.2. There exist $r-s+1$ linearly independent highest weight vectors in $\mathcal{B}$ with highest weight $(r, s, 0, \cdots, 0)$ for all $r, s \in \mathbb{N}$ satisfying $r \geq s$.

Proof: Let $x=u_{2 n-1}^{1}, y=u_{2 n-1}^{2 n}, z=u_{2 n}^{1}$ and $w=u_{2 n}^{2 n}$. Let $p=r-s$. It is easy to see that

$$
E_{i}(x)=E_{i}(y)=E_{i}(z)=E_{i}(w)=0 \quad \text { for } \quad i>1 .
$$

Also,

$$
E_{1}(x)=-z, E_{1}(y)=-w, E_{1}(z)=E_{2}(w)=0 .
$$

Further,

$$
\begin{gathered}
K_{1}(x)=q^{-1} x, K_{1}(y)=q^{-1} y, K_{1}(z)=q z, K_{1}(w)=q w \\
K_{2}(x)=q x, K_{2}(y)=q y, K_{2}(z)=z, K_{2}(w)=w .
\end{gathered}
$$

and for $i>2, K_{i}$ fixes these elements. Now, using the relations (2.1) and the facts that $\Delta\left(E_{1}\right)=E_{1} \otimes K_{1}+1 \otimes E_{1}$ and $\Delta\left(K_{i}\right)=K_{i} \otimes K_{i}$, we get

$$
\begin{aligned}
E_{1}\left(y^{s} z^{s}\right) & =C_{1}^{0} y^{s-1} z^{s} w . \\
E_{1}\left(x y^{s-1} z^{s-1} w\right)= & C_{1}^{1} y^{s-1} z^{s} w+C_{2}^{1} x y^{s-2} z^{s-1} w . \\
\cdots & \cdots \\
E_{1}\left(x^{s-1} y z w^{s-1}\right)= & C_{1}^{s-1} x^{s-2} y z^{2} w^{s-1}+C_{2}^{s-1} x^{s-1} z w^{s} . \\
E_{1}\left(x^{s} w^{s}\right)= & C_{1}^{s} x^{s-1} z w^{s}
\end{aligned}
$$

where $C_{i}^{j}$ 's are nonzero constants. This shows that we can choose nonzero constants $c_{1} \cdots c_{s}$ such that $E_{1}\left(y^{s} z^{s}+c_{1} x y^{s-1} z^{s-1} w+\cdots+c_{s} x^{s} w^{s}\right)=0$. Let $\omega=s_{1} s_{2} \cdots s_{n-1} s_{n} s_{n-1} \cdots s_{1}$ and 
$\omega^{\prime}=s_{1} s_{2} \cdots s_{n-1} s_{n} s_{n-1} \cdots s_{2}$. Let $\pi_{\omega}$ and $\pi_{\omega^{\prime}}$ be the representations of $C\left(S P_{q}(2 n)\right)$ as defined in subsection (2.2). It is easy to see that $\pi_{\omega^{\prime}}(z)=0$. Hence,

$\pi_{\omega^{\prime}}\left(y^{s} z^{s}+c_{1} x y^{s-1} z^{s-1} w+\cdots+c_{s} x^{s} w^{s}\right)\left(e_{0} \otimes e_{0} \otimes \cdots \otimes e_{0}\right)=\pi_{\omega^{\prime}}\left(c_{s} x^{s} w^{s}\right)\left(e_{0} \otimes e_{0} \otimes \cdots \otimes e_{0}\right) \neq 0$

which shows that $y^{s} z^{s}+c_{1} x y^{s-1} z^{s-1} w+\cdots+c_{s} x^{s} w^{s} \neq 0$. Since $\omega^{\prime}$ is a subword of $\omega, \pi_{\omega^{\prime}}$ factors through $\pi_{\omega}$ which implies that $\pi_{\omega}\left(y^{s} z^{s}+c_{1} x y^{s-1} z^{s-1} w+\cdots+c_{s} x^{s} w^{s}\right) \neq 0$.

Define $b_{j}=z^{j} w^{p-j}\left(y^{s} z^{s}+c_{1} x y^{s-1} z^{s-1} w+\cdots+c_{s} x^{s} w^{s}\right)$ for $j \in\{0, \cdots, p\}$. Clearly, $b_{j} \in \mathcal{B}$. One can directly verify that $b_{j}$ are elements with highest weight $(r, s, 0, \cdots, 0)$. Now, look at the $(n)^{\text {th }}$ position of $\pi_{\omega}\left(b_{j}\right)\left(e_{0} \otimes e_{0} \otimes \cdots e_{0}\right)$. One term has $e_{p-j}$ at $(n)^{\text {th }}$ position and other terms have $e_{\ell}$ at $(n)^{\text {th }}$ position where $\ell<p-j$ (see diagram 4 ). This proves that $b_{j}$ are linearly independent.

Proof: (of theorem 3.1) One can easily check that $u_{m}^{2 n}$ and $u_{m}^{1}=\epsilon_{m} q^{\rho_{1}-\rho_{m}}\left(u_{2 n-m+1}^{2 n}\right)^{*}$ are in $C\left(S P_{q}(2 n) / S P_{q}(2 n-2)\right)$ for $m=1,2, \cdots 2 n$. So,

$$
C\left(S P_{q}(2 n) / S P_{q}(2 n-2)\right) \supseteq C^{*}\left\{u_{m}^{1}, u_{m}^{2 n}: j \in\{1,2, \cdots 2 n\}\right\} .
$$

To show the equality, consider the co-multiplication action on $C\left(S P_{q}(2 n) / S P_{q}(2 n-2)\right)$ by the compact quantum group $C\left(S P_{q}(2 n)\right)$,

$$
\begin{aligned}
C\left(S P_{q}(2 n) / S P_{q}(2 n-2)\right. & \longrightarrow C\left(S P_{q}(2 n) / S P_{q}(2 n-2) \otimes C\left(S P_{q}(2 n)\right)\right. \\
a & \longmapsto \Delta a
\end{aligned}
$$

By theorem 1.5, Podles [15], we get,

$$
C\left(S P_{q}(2 n) / S P_{q}(2 n-2)=\overline{\oplus_{\lambda \in \widehat{S P(2 n)}} \oplus_{i \in I_{\lambda}} W_{\lambda, i}}\right.
$$

where $\lambda$ represents a finite-dimensional irreducible co-representation $u^{\lambda}$ of $C\left(S P_{q}(2 n)\right), W_{\lambda, i}$ corresponds to $u^{\lambda}$ for all $i \in I_{\lambda}$ and $I_{\lambda}$ is the multiplicity of $u^{\lambda}$. Define

$$
\mathcal{A}=\oplus_{\lambda \in \widehat{S P(2 n)}} \oplus_{i \in I_{\lambda}} W_{\lambda, i}
$$

We will prove that $\mathcal{A} \subseteq C^{*}\left\{u_{m}^{1}, u_{m}^{2 n}: m \in\{1,2, \cdots 2 n\}\right\}$ which will suffice to show the claim.

The finite-dimensional irreducible co-representations of $C\left(S P_{q}(2 n)\right)$ or equivalently irreducible representations of $U_{q}(s p(2 n))$ are in a one-to-one correspondence with $n$-tuples of integers $\lambda=\left(\lambda_{1}, \cdots, \lambda_{n}\right)$ satisfying the inequalities

$$
\lambda_{1} \geq \lambda_{2} \geq \cdots \geq \lambda_{n} \geq 0
$$

Such an $n$-tuple $\lambda$ is called the highest weight of the corresponding representation which we denote by $V(\lambda)$. The restriction of $V(\lambda)$ to the subalgebra $U_{q}(s p(2 n-2))$ is isomorphic to a direct sum of irreducible finite-dimensional representations $V^{\prime}(\mu), \mu=\left(\mu_{1}, \cdots, \mu_{n-1}\right)$ of 
$U_{q}(\operatorname{sp}(2 n-2))$ with certain multiplicity $n_{\lambda}(\mu)$. The multiplicity $n_{\lambda}(\mu)$ is equal to the number of $n$-tuples of integers $\left(\nu_{1}, \cdots \nu_{n}\right)$ satisfying the inequalities,

$$
\begin{aligned}
& \lambda_{1} \geq \nu_{1} \geq \lambda_{2} \geq \nu_{2} \geq \cdots \geq \lambda_{n} \geq \nu_{n} \geq 0 . \\
& \nu_{1} \geq \mu_{1} \geq \nu_{2} \geq \mu_{2} \cdots \geq \mu_{n-1} \geq \nu_{n} \geq 0 .
\end{aligned}
$$

We refer to Zhelobenko [21] for more detail. Now, this shows that a finite-dimensional irreducible representation of $U_{q}(s p(2 n))$ with highest weight $\lambda$ when restricted to the sublagebra $U_{q}(s p(2 n-2))$ contains trivial representation if and only if $\lambda_{i}=0$ for all $i \geq 3$ and for such $\lambda$, the multiplicity of trivial representation denoted by $n_{\lambda}(0)=\lambda_{1}-\lambda_{2}+1$. By theorem 1.7 , Podles [15],

$$
I_{\lambda}=n_{\lambda}(0)= \begin{cases}\lambda_{1}-\lambda_{2}+1, & \text { if } \lambda_{i}=0 \text { for all } i \geq 3, \\ 0, & \text { otherwise }\end{cases}
$$

It follows from proposition 3.2 that $\mathcal{B} \subseteq C^{*}\left\{u_{m}^{1}, u_{m}^{2 n}: m \in\{1,2, \cdots 2 n\}\right\}$ contains $\lambda_{1}-\lambda_{2}+1$ linearly independent highest weight vector with highest weight $\left(\lambda_{1}, \lambda_{2}, 0, \cdots, 0\right)$. This proves that for each co-representation $\lambda$ of $S P(2 n), \oplus_{i \in I_{\lambda}} W_{\lambda, i} \subseteq C^{*}\left\{u_{m}^{1}, u_{m}^{2 n}: m \in\{1,2, \cdots 2 n\}\right\}$ which further shows that $\mathcal{A} \subseteq C^{*}\left\{u_{m}^{1}, u_{m}^{2 n}: m \in\{1,2, \cdots 2 n\}\right\}$. This proves the claim.

\section{Quantum quaternion sphere}

Our main aim in this section is to describe $C\left(S P_{q}(2 n) / S P_{q}(2 n-2)\right)$ as a universal $C^{*}$-algebra given by a finite set of generators and relations.

Definition 4.1. We define $C^{*}$-algebra $C\left(H_{q}^{2 n}\right)$ of continuous functions on the quantum quaternion sphere as the universal $C^{*}$-algebra generated by elements $z_{1}, z_{2}, \ldots . z_{2 n}$ satisfying the following relations

$$
\begin{aligned}
z_{i} z_{j} & =q z_{j} z_{i} & & \text { for } i>j, i+j \neq 2 n+1 \\
z_{i} z_{i^{\prime}} & =q^{2} z_{i^{\prime}} z_{i}-\left(1-q^{2}\right) \sum_{k>i} q^{i-k} z_{k} z_{k^{\prime}} & & \text { for } i>n \\
z_{i}^{*} z_{i^{\prime}} & =q^{2} z_{i^{\prime}} z_{i}^{*} & & \\
z_{i}^{*} z_{j} & =q z_{j} z_{i}^{*} & & \text { for } i+j>2 n+1, i \neq j \\
z_{i}^{*} z_{j} & =q z_{j} z_{i}^{*}+\left(1-q^{2}\right) \epsilon_{i} \epsilon_{j} q^{\rho_{i}+\rho_{j}} z_{i^{\prime}} z_{j^{\prime}}^{*} & & \text { for } i+j<2 n+1, i \neq j \\
z_{i}^{*} z_{i} & =z_{i} z_{i}^{*}+\left(1-q^{2}\right) \sum_{k>i} z_{k} z_{k}^{*} & & \text { for } i>n \\
z_{i}^{*} z_{i} & =z_{i} z_{i}^{*}+\left(1-q^{2}\right) q^{2 \rho_{i}} z_{i^{\prime}} z_{i^{\prime}}^{*}+\left(1-q^{2}\right) \sum_{k>i} z_{k} z_{k}^{*} & & \text { for } i \leq n \\
\sum_{i=1}^{2 n} z_{i} z_{i}^{*} & =1 & &
\end{aligned}
$$


In what follows, we will find a faithful realization of this $C^{*}$-algebra on a Hilbert space. For this, we will first find all irreducible representations of the above $C^{*}$-algebra.

It follows from the commutation relations that $\left\|z_{i}\right\| \leq 1$, for $1 \leq i \leq 2 n$ and $z_{2 n}$ is normal. We denote $z_{2 n}^{*} z_{2 n}$ by $\omega$. Using the relations (4.1), (4.3) and (4.5), we have

$$
\begin{array}{ll}
z_{i} \omega=q^{-2} \omega z_{i}, & z_{i}^{*} \omega=q^{2} \omega z_{i}^{*} \quad \text { for all } i \notin\{1,2 n\}, \\
z_{1} \omega=q^{-4} \omega z_{1}, & z_{1}^{*} \omega=q^{4} \omega z_{1}^{*} .
\end{array}
$$

Proposition 4.2. Let $\pi$ be a representation of $C\left(H_{q}^{2 n}\right)$. Then one has

1. $\pi(\omega)=I \quad$ on $\bigcap_{i=1}^{2 n-1} \operatorname{ker} \pi\left(z_{i}^{*}\right)$,

2. $1_{\left(q^{2 m+2}, q^{2 m}\right)}(\pi(\omega))=0 \quad \forall m \in \mathbb{N}$,

3. $\operatorname{ker}\left(\pi\left(z_{i}\right)\right) \subseteq \operatorname{ker}\left(\pi\left(z_{k}^{*}\right)\right)$ for $k \geq i$ and $1 \leq i \leq 2 n$,

4. if $u$ is a nonzero eigenvector of $\pi(\omega)$ corresponding to the eigenvalue $q^{2 m}$, then $u \notin$ $\operatorname{ker} \pi\left(z_{i}\right)$ for $1 \leq i \leq 2 n-1$,

5. either $\sigma(\pi(\omega))=\left\{q^{2 m}: m \in \mathbb{N}\right\} \bigcup\{0\}$ or $\sigma(\pi(\omega))=\{0\}$.

Proof:

1. Easy to see from (4.8).

2. From the commutation relations, it follows that $z_{1}^{*} f(\omega)=f\left(q^{4} \omega\right) z_{1}^{*}$ and $z_{i}^{*} f(\omega)=$ $f\left(q^{2} \omega\right) z_{i}^{*}$ for all $i \neq 1$ for all continuous functions $f$ and hence for all $L_{\infty}$ functions. Thus

$$
\begin{aligned}
\pi\left(z_{1}\right)^{*} 1_{\left(q^{2 n+2}, q^{2 n}\right)}(\pi(\omega)) & =1_{\left(q^{2 n+2}, q^{2 n}\right)}\left(q^{4} \pi(\omega)\right) \pi\left(z_{1}\right)^{*} \\
& =1_{\left(q^{2 n-2}, q^{2 n-4}\right)}(\pi(\omega)) \pi\left(z_{1}\right)^{*} \\
\pi\left(z_{i}\right)^{*} 1_{\left(q^{2 n+2}, q^{2 n}\right)}(\pi(\omega)) & =1_{\left(q^{2 n+2}, q^{2 n}\right)}\left(q^{2} \pi(\omega)\right) \pi\left(z_{i}\right)^{*} \\
& =1_{\left(q^{2 n}, q^{2 n-2}\right)}(\pi(\omega)) \pi\left(z_{1}\right)^{*} .
\end{aligned}
$$

By repeated application and using (4.8) and the fact that $\sigma(\omega) \subseteq[0,1]$, it follows that $1_{\left(q^{2 n+2}, q^{2 n}\right)}(\pi(\omega))=0$.

3. Let $h \in \operatorname{ker}\left(\pi\left(z_{i}\right)\right)$ and $i>n$. Using (4.6), we have

$$
\left\langle z_{i}^{*} z_{i} h, h\right\rangle=\left\langle z_{i} z_{i}^{*} h+\left(1-q^{2}\right) \sum_{k>i} z_{k} z_{k}^{*} h, h\right\rangle .
$$

Therefore it follows that

$$
\left\|z_{i}^{*} h\right\|^{2}+\left(1-q^{2}\right) \sum_{k>i}\left\|z_{k}^{*} h\right\|=0
$$

Hence $\left\|z_{k}^{*} h\right\|=0$ for all $k \geq i$, which means $h \in \operatorname{ker} \pi\left(z_{k}^{*}\right)$ or all $k \geq i$. For $i \leq n$, use (4.7) and follow similar steps. 
4. From part 3, we have $\operatorname{ker}\left(z_{i}\right) \subseteq \operatorname{ker}\left(z_{2 n}^{*}\right)=\operatorname{ker}\left(z_{2 n}\right)=\operatorname{ker}(\omega)$. Now if $u$ is a non-zero eigenvector of $\pi(\omega)$ corresponding to eigenvalue $q^{2 m}$ for some $m \in \mathbb{N}$, then $u \notin \operatorname{ker}\left(z_{2 n}^{*}\right)$. Hence $u \notin \operatorname{ker}\left(z_{i}\right)$ for $1 \leq i \leq 2 n$.

5. From part 2 and the fact that $\|\omega\| \leq 1$, it follows that $\sigma(\pi(\omega)) \subseteq\left\{q^{2 m}: m \in \mathbb{N}\right\} \bigcup\{0\}$. Define

$$
A=\left\{m \in \mathbb{N}: q^{2 m} \in \sigma(\pi(\omega))\right\} .
$$

If $A=\emptyset$, we have $\sigma(\pi(\omega))=\{0\}$. If $A \neq \emptyset$, define

$$
m_{0}=\inf \left\{m \in \mathbb{N}: q^{2 m} \in \sigma(\pi(\omega))\right\} .
$$

Let $u$ be a nonzero eigenvector corresponding to $q^{2 m_{0}}$. Assume $u \notin \operatorname{ker} \pi\left(z_{i}^{*}\right)$ for some $i \in\{1,2, \cdots, 2 n-1\}$. Then from (4.1), it follows that $\pi\left(z_{i}^{*}\right) u$ is a nonzero eigenvector corresponding to the eigenvalue $q^{2 m_{0}-2}$ or $q^{2 m_{0}-4}$ depending on whether $i \neq 1$ or $i=1$, which contradicts the fact that $m_{0}$ is $\inf A$. Hence $u \in \bigcap_{i=1}^{2 n-1} \operatorname{ker} \pi\left(z_{i}^{*}\right)$. As $\pi(\omega)=I$ on $\bigcap_{i=1}^{2 n-1} \operatorname{ker} \pi\left(z_{i}^{*}\right)$, we get $m_{0}=0$. From part 4 , it follows that $u \notin \operatorname{ker} \pi\left(z_{i}\right)$ for any $i \in\{1,2, \cdots 2 n\}$. Now applying (4.1), we have $\pi\left(z_{2}^{m}\right) u$ is a nonzero eigenvector corresponding to eigenvalue $q^{2 m}$ for all $m \in \mathbb{N}$. This proves the claim.

Let $\pi$ be a representation of $C\left(H_{q}^{2 n}\right)$ in a Hilbert space $\mathcal{H}$. From (4.1), it follows that $\operatorname{ker}(\pi(\omega))$ is an invariant subspace for $\pi$. Therefore if $\pi$ is irreducible, then either $\pi(\omega)=0$ or $\operatorname{ker}(\pi(\omega))=0$. Assume $\pi(\omega) \neq 0$. Then $\operatorname{ker}(\pi(\omega))=0$, and by part 5 , we have $\sigma(\pi(\omega))=$ $\left\{q^{2 m}: m \in \mathbb{N}\right\} \bigcup\{0\}$. Hence $\mathcal{H}$ decomposes as

$$
\mathcal{H}=\oplus_{m \in \mathbb{N}} \mathcal{H}_{m}
$$

where $\mathcal{H}_{m}$ is the eigenspace of $\pi(\omega)$ corresponding to the eigenvalue $q^{2 m}$. It is clear from (4.1) that for $1<i<2 n$, the operator $\pi\left(z_{i}\right)$ sends $\mathcal{H}_{m}$ into $\mathcal{H}_{m+1}$ and $\pi\left(z_{i}^{*}\right)$ sends $\mathcal{H}_{m}$ into $\mathcal{H}_{m-1}$, $\pi\left(z_{1}\right)$ sends $\mathcal{H}_{m}$ into $\mathcal{H}_{m+2}$ and $\pi\left(z_{1}^{*}\right)$ sends $\mathcal{H}_{m}$ into $\mathcal{H}_{m-2}$. Also, both $\pi\left(z_{2 n}\right)$ and $\pi\left(z_{2 n}^{*}\right)$ keep $\mathcal{H}_{m}$ invariant. Observe that $\left.\pi\left(z_{2 n}\right)\right|_{\mathcal{H}_{0}}$ is an unitary operator.

Proposition 4.3. Let $u \in \bigcap_{i=1}^{2 n-1} \operatorname{ker} \pi\left(z_{i}^{*}\right)$. Then

$$
\pi\left(z_{2 n}\right) u \in \bigcap_{i=1}^{2 n-1} \operatorname{ker} \pi\left(z_{i}^{*}\right), \quad \pi\left(z_{2 n}^{*}\right) u \in \bigcap_{i=1}^{2 n-1} \operatorname{ker} \pi\left(z_{i}^{*}\right) .
$$

Proof: We need to show that $\pi\left(z_{i}\right) \pi\left(z_{2 n}\right) u=\pi\left(z_{i}^{*}\right) \pi\left(z_{2 n}\right) u=0$ for all $i \in\{1,2, \cdots 2 n-1\}$, which follows from (4.1), (4.3) and (4.4).

Let $K$ be a subspace of $\cap_{i=1}^{2 n-1} \operatorname{ker} \pi\left(z_{i}^{*}\right)$ invariant under the $C^{*}$-algebra generated by $\pi\left(z_{2 n}\right)$. Define

$$
\mathcal{H}^{K}=\text { linear span }\left\{\pi\left(z_{1}\right)^{\alpha_{1}} \pi\left(z_{2}\right)^{\alpha_{2}} \cdots \pi\left(z_{2 n-1}\right)^{\alpha_{2 n-1}} h: h \in K\right\} \text {. }
$$


Lemma 4.4. Let $\pi$ be an irreducible representation of $C\left(H_{q}^{2 n}\right)$ such that $\pi\left(z_{2 n}\right) \neq 0$. Then $\mathcal{H}^{K}$ is an invariant subspace of $\pi$.

Proof: Let $h \in K$. Define

$$
h\left(\alpha_{1}, \alpha_{2}, \cdots, \alpha_{2 n-1}\right)=\pi\left(z_{1}\right)^{\alpha_{1}} \pi\left(z_{2}\right)^{\alpha_{2}} \cdots \pi\left(z_{2 n-1}\right)^{\alpha_{2 n-1}} h .
$$

It is clear that $\pi\left(z_{2 n}\right)$ keeps $\mathcal{H}^{K}$ invariant, as

$$
\pi\left(z_{2 n}\right) h\left(\alpha_{1}, \alpha_{2}, \cdots, \alpha_{2 n-1}\right)=q^{\left(\sum_{l=1}^{2 n-1} \alpha_{l}\right)+\alpha_{1}} h\left(\alpha_{1}, \alpha_{2}, \cdots, \alpha_{2 n-1}\right) .
$$

For $1 \leq i \leq n$,

$$
\pi\left(z_{i}\right) h\left(\alpha_{1}, \alpha_{2}, \cdots, \alpha_{2 n-1}\right)=q^{\sum_{l=1}^{i-1} \alpha_{l}} h\left(\alpha_{1}, \cdots, \alpha_{i-1}, \alpha_{i}+1, \alpha_{i+1}, \cdots, \alpha_{2 n-1}\right) \in \mathcal{H}^{K} .
$$

For $i=2 n-1$,

$$
\pi\left(z_{2 n-1}\right) h\left(\alpha_{1}, \alpha_{2}, \cdots, \alpha_{2 n-1}\right)=q^{\alpha_{1}} \pi\left(z_{1}\right)^{\alpha_{1}} \pi\left(z_{2 n-1}\right) \pi\left(z_{2}\right)^{\alpha_{2}} \pi\left(z_{3}\right)^{\alpha_{3}} \cdots \pi\left(z_{2 n-1}\right)^{\alpha_{2 n-1}} h .
$$

Repeated application of (4.2) gives

$$
z_{i} z_{i^{\prime}}^{m}=q^{2 m} z_{i^{\prime}}^{m} z_{i}-\left(1-q^{2 m}\right) \sum_{k>i} q^{i-k} z_{i^{\prime}}^{m-1} z_{k} z_{k^{\prime}}
$$

Hence we have

$$
\begin{aligned}
& \pi\left(z_{2 n-1}\right) h\left(\alpha_{1}, \alpha_{2}, \cdots, \alpha_{2 n-1}\right) \\
& =q^{\alpha_{1}} \pi\left(z_{1}\right)^{\alpha_{1}} \pi\left(q^{2 \alpha_{2}} z_{2}^{\alpha_{2}} z_{n-1}-q\left(1-q^{2 \alpha_{2}}\right) z_{2}^{\alpha_{2}-1} z_{2 n} z_{1}\right) \pi\left(z_{3}\right)^{\alpha_{3}} \cdots \pi\left(z_{2 n-1}\right)^{\alpha_{2 n-1}} h . \\
& =q^{\alpha_{1}+2 \alpha_{2}+\sum_{l=3}^{2 n-3} h\left(\alpha_{1}, \alpha_{2}, \cdots, \alpha_{2 n-1}+1\right)} \\
& \quad-q^{\alpha_{1}+1}\left(1-q^{2 \alpha_{2}}\right) \pi\left(z_{1}\right)^{\alpha_{1}} \pi\left(z_{2}^{\alpha_{2}-1} z_{2 n} z_{1}\right) h\left(0,0, \alpha_{3}, \cdots, \alpha_{2 n-1}\right) .
\end{aligned}
$$

We have shown above that $\pi\left(z_{1}\right), \pi\left(z_{2}\right)$ and $\pi\left(z_{2 n}\right)$ keep $\mathcal{H}^{K}$ invariant. Hence

$$
\pi\left(z_{2 n-1}\right) h\left(\alpha_{1}, \alpha_{2}, \cdots, \alpha_{2 n-1}\right) \in \mathcal{H}^{K}
$$

Similarly, by using backward induction we can show that $\mathcal{H}^{K}$ is invariant under the actions of $\pi\left(z_{1}\right), \pi\left(z_{2}\right), \cdots \pi\left(z_{n}\right)$. Also, we have

$$
\pi\left(z_{2 n}^{*}\right) h\left(\alpha_{1}, \alpha_{2}, \cdots, \alpha_{2 n-1}\right)=q^{\left(\sum_{l=1}^{2 n-1} \alpha_{l}\right)+\alpha_{1}} h\left(\alpha_{1}, \alpha_{2}, \cdots, \alpha_{2 n-1}\right) .
$$

This shows that $\pi\left(z_{2 n}^{*}\right)$ keeps $\mathcal{H}^{K}$ invariant. By applying (4.5) and (4.6) repeatedly, we get

$$
\begin{aligned}
z_{2 n-1}^{*} z_{1}^{m} & =q^{m} z_{1}^{m} z_{2 n-1}^{*}+m q^{m}\left(1-q^{2}\right) \epsilon_{2 n-1} \epsilon_{1} q^{\rho_{2 n-1}+\rho_{1}} z_{1}^{m-1} z_{2} z_{2 n}^{*}, \\
z_{2 n-1}^{*} z_{2 n-1}^{m} & =z_{2 n-1}^{m} z_{2 n-1}^{*}+\left(1-q^{2 m}\right) z_{2 n-1}^{m-1} \omega .
\end{aligned}
$$


Hence we have

$$
\begin{aligned}
\pi & \left(z_{2 n-1}^{*}\right) h\left(\alpha_{1}, \alpha_{2}, \cdots, \alpha_{2 n-1}\right) \\
= & q^{\alpha_{1}} \pi\left(z_{1}^{\alpha_{1}} z_{2 n-1}^{*}+\alpha_{1} q^{\alpha_{1}}\left(1-q^{2}\right) \epsilon_{2 n-1} \epsilon_{1} q^{\rho_{2 n-1}+\rho_{1}} z_{1}^{\alpha_{1}-1} z_{2} z_{2 n}^{*}\right) \pi\left(z_{2}\right)^{\alpha_{2}} \cdots \pi\left(z_{2 n-1}\right)^{\alpha_{2 n-1}} h \\
= & q^{\left(\sum_{l=1}^{2 n-2} l\right)+\alpha_{2}} \pi\left(z_{1}\right)^{\alpha_{1}} \cdots \pi\left(z_{2 n-2}\right)^{\alpha_{2 n-2}} \pi\left(z_{2 n-1}\right)^{*} \pi\left(z_{2 n-1}\right)^{\alpha_{2 n-1}} h \\
& -\alpha_{1} q^{\alpha_{1}}\left(1-q^{2}\right) q^{\rho_{2 n-1}+\rho_{1}} \pi\left(z_{1}^{\alpha_{1}-1}\right) \pi\left(z_{2}\right) \pi\left(z_{2 n}^{*}\right) \pi\left(z_{2}\right)^{\alpha_{2}} \cdots \pi\left(z_{2 n-1}\right)^{\alpha_{2 n-1}} h \\
= & \left(1-q^{2 \alpha_{1}}\right) q^{\left(\sum_{l=1}^{2 n-2} l\right)+\alpha_{2}} \pi\left(z_{1}\right)^{\alpha_{1}} \cdots \pi\left(z_{2 n-2}\right)^{\alpha_{2 n-2}} \pi\left(z_{2 n-1}\right)^{\alpha_{2 n-1}-1} \pi(\omega) h \\
& -\alpha_{1} q^{\alpha_{1}}\left(1-q^{2}\right) q^{\rho_{2 n-1}+\rho_{1}} \pi\left(z_{1}^{\alpha_{1}-1}\right) \pi\left(z_{2}\right) \pi\left(z_{2 n}^{*}\right) \pi\left(z_{2}\right)^{\alpha_{2}} \cdots \pi\left(z_{2 n-1}\right)^{\alpha_{2 n-1}} h .
\end{aligned}
$$

Since $\pi\left(z_{1}\right), \pi\left(z_{2}\right)$ and $\pi\left(z_{2 n}^{*}\right)$ keep $\mathcal{H}^{K}$ invariant, $\mathcal{H}^{K}$ is invariant under the action of $\pi\left(z_{2 n-1}^{*}\right)$. By using backward induction and following similar steps, we can show that $\mathcal{H}^{K}$ is invariant for $\pi$.

It follows from the lemma above that if $K$ is an invariant subspace for $\bigcap_{i=1}^{2 n-1} \operatorname{ker} \pi\left(z_{i}^{*}\right)$, then $\mathcal{H}^{K}$ is an invariant subspace for $\pi$ and is a proper invariant subspace for $\pi$ if $K$ is a proper subspace of $\bigcap_{i=1}^{2 n-1} \operatorname{ker} \pi\left(z_{i}^{*}\right)$. Therefore, if $\pi$ is an irreducible representation, then the space $\bigcap_{i=1}^{2 n-1} \operatorname{ker} \pi\left(z_{i}^{*}\right)$ is one dimensional.

Corollary 4.5. Let $\pi$ be an irreducible representation such that $\pi\left(z_{2 n}\right) \neq 0$. Let $u$ be a unit vector in $\bigcap_{i=1}^{2 n-1} \operatorname{ker} \pi\left(z_{i}^{*}\right)$. Then

$$
\mathcal{H}_{m}=\text { linear span }\left\{\pi\left(z_{1}\right)^{\alpha_{1}} \pi\left(z_{2}\right)^{\alpha_{2}} \cdots \pi\left(z_{2 n-1}\right)^{\alpha_{2 n-1}} u:\left(\sum_{l=1}^{2 n-1} \alpha_{l}\right)+\alpha_{1}=m\right\} .
$$

Proof: It follows from Lemma 4.4, equation (4.9) and equation (4.10).

Define

$$
u_{\alpha_{2}, \cdots, \alpha_{2 n-1}, \alpha_{0}}=\pi\left(z_{2 n-1}\right)^{\alpha_{2 n-1}} \pi\left(z_{2 n-2}\right)^{\alpha_{2 n-2}} \cdots \pi\left(z_{2}\right)^{\alpha_{2}}\left[\pi\left(z_{n}\right), \pi\left(z_{n+1}\right)\right]^{\alpha_{0}} u
$$

where $\alpha_{i} \in \mathbb{N}$ and $u$ is as in Corollary 4.5. Now we develop some tools by analyzing the defining relations more closely.

Proposition 4.6. Let $\pi$ be an irreducible representation of $C\left(H_{q}^{2 n}\right)$ such that $\pi\left(z_{2 n}\right) \neq 0$. Then 1. for $i>n$,

$$
\pi\left(z_{i}\right)^{*} \pi\left(z_{i}\right)^{m}=\pi\left(z_{i}\right)^{m} \pi\left(z_{i}\right)^{*}+\left(1-q^{2 m}\right) \sum_{k>i} \pi\left(z_{i}\right)^{m-1} \pi\left(z_{k}\right) \pi\left(z_{k}\right)^{*}
$$

2. for $i \leq n$,

$$
\begin{aligned}
\pi\left(z_{i}\right)^{*} \pi\left(z_{i}\right)^{m}= & \pi\left(z_{i}\right)^{m} \pi\left(z_{i}\right)^{*}+q^{2 \rho_{i}}\left(1-q^{2 m}\right) \pi\left(z_{i}\right)^{m-1} \pi\left(z_{i^{\prime}}\right) \pi\left(z_{i^{\prime}}\right)^{*} \\
& +\left(1-q^{2 m}\right) \sum_{k>i} \pi\left(z_{i}\right)^{m-1} \pi\left(z_{k}\right) \pi\left(z_{k}\right)^{*}
\end{aligned}
$$


3. for $i+j<2 n+1, i \neq j$,

$$
\pi\left(z_{i}\right)^{*} \pi\left(z_{j}\right)^{m}=q^{m} \pi\left(z_{j}\right)^{m} \pi\left(z_{i}\right)^{*}+m q^{m}\left(1-q^{2}\right) \epsilon_{i} \epsilon_{j} q^{\rho_{i}+\rho_{j}} \pi\left(z_{j}\right)^{m-1} \pi\left(z_{i^{\prime}}\right) \pi\left(z_{j^{\prime}}\right)^{*}
$$

4. for $i>n$,

$$
\pi\left(z_{i}\right)^{*}\left[\pi\left(z_{n}\right), \pi\left(z_{n+1}\right)\right]^{m}=q^{2 m}\left[\pi\left(z_{n}\right), \pi\left(z_{n+1}\right)\right]^{m} \pi\left(z_{i}\right)^{*}
$$

5. for $i>n$,

$$
\pi\left(z_{i}\right)^{*} u_{\alpha_{2}, \cdots, \alpha_{2 n-1}, \alpha_{0}}=C u_{\alpha_{2}, \cdots \alpha_{i-1}, \alpha_{i}-1, \alpha_{i+1} \cdots, \alpha_{2 n-1}, \alpha_{0}}
$$

where $C$ is some non-zero constant;

6. for $n<i<2 n$,

$$
\pi\left(z_{i}\right)^{*} u_{\alpha_{2}, \cdots \alpha_{i-1}, 0, \alpha_{i+1} \cdots, \alpha_{2 n-1}, \alpha_{0}}=0
$$

7. we have

$$
\begin{aligned}
& \pi\left(z_{n}\right)^{*}\left[\pi\left(z_{n}\right), \pi\left(z_{n+1}\right)\right]^{m}=q^{2 m}\left[\pi\left(z_{n}\right), \pi\left(z_{n+1}\right)\right]^{m} \pi\left(z_{n}\right)^{*}+\left(1-q^{4}\right)\left(1-q^{2}\right) \times \\
& \left(\sum_{l=0}^{k-1} q^{4 l}\left[\pi\left(z_{n}\right), \pi\left(z_{n+1}\right)\right]^{k-1-l} \pi\left(z_{n+1}\right)\left[\pi\left(z_{n}\right), \pi\left(z_{n+1}\right)\right]^{l}\right) \sum_{k>n+1} \pi\left(z_{k}\right) \pi\left(z_{k}\right)^{*}
\end{aligned}
$$

8. for $1<i \leq n$,

$$
\begin{aligned}
& \pi\left(z_{i}\right)^{*} \pi\left(z_{i-1}\right)^{\alpha_{i-1}} \cdots \pi\left(z_{2}\right)^{\alpha_{2}}\left[\pi\left(z_{n}\right), \pi\left(z_{n+1}\right)\right]^{\alpha_{0}} u \\
& =C \pi\left(z_{i-1}\right)^{\alpha_{i-1}} \cdots \pi\left(z_{2}\right)^{\alpha_{2}} \pi\left(z_{i}\right)^{*}\left[\pi\left(z_{n}\right), \pi\left(z_{n+1}\right)\right]^{\alpha_{0}} u
\end{aligned}
$$

where $C$ is some non-zero constant;

9. we have

$$
\left[\pi\left(z_{n}\right), \pi\left(z_{n+1}\right)\right]^{*}\left[\pi\left(z_{n}\right), \pi\left(z_{n+1}\right)\right]^{m} u=C\left[\pi\left(z_{n}\right), \pi\left(z_{n+1}\right)\right]^{m-1} u,
$$

where $C$ is some non-zero constant;

10. for $1<i \leq n$,

$$
\pi\left(z_{i}\right) \pi\left(z_{i}^{\prime m}\right)=q^{2 m} \pi\left(z_{i^{\prime}}^{m}\right) \pi\left(z_{i}\right)-\sum_{k>i}\left(1-q^{2 m}\right) q^{i-k} \pi\left(z_{k}\right) \pi\left(z_{k^{\prime}}\right) \pi\left(z_{i}^{m-1}\right)
$$

11. for $1 \leq i<n$,

$$
\pi\left(z_{i}^{*}\right)\left[\pi\left(z_{n}\right), \pi\left(z_{n+1}\right)\right]^{m} u=C \pi\left(z_{i}^{\prime}\right)\left[\pi\left(z_{n}\right), \pi\left(z_{n+1}\right)\right]^{m-1} u,
$$

where $C$ is some constant;

12. we have

$$
\pi\left(z_{1}\right)^{*} u_{\alpha_{2}, \cdots, \alpha_{n}, 0, \cdots, 0, \alpha_{0}}=C u_{\alpha_{2}, \cdots \alpha_{n}, 0, \cdots, 0, \alpha_{0}-1} .
$$

Proof: We will prove part 4 and part 9 of this proposition. Other parts will follow by direct calculation using the commutation relations. 
1. For $i>n+1$, it follows from (4.1). For $i=n+1$, it is enough to show for $m=1$.

$$
\begin{aligned}
\pi\left(z_{n+1}\right)^{*}\left[\pi\left(z_{n}\right), \pi\left(z_{n+1}\right)\right] \\
=\pi\left(z_{n+1}\right)^{*} \pi\left(z_{n}\right) \pi\left(z_{n+1}\right)-\pi\left(z_{n+1}\right)^{*} \pi\left(z_{n+1}\right) \pi\left(z_{n}\right) \\
=q^{2} \pi\left(z_{n}\right) \pi\left(z_{n+1}\right)^{*} \pi\left(z_{n+1}\right)-\pi\left(z_{n+1}\right) \pi\left(z_{n+1}\right)^{*} \pi\left(z_{n}\right) \\
\quad-\left(1-q^{2}\right) \sum_{k>n+1} \pi\left(z_{k}\right) \pi\left(z_{k}^{*}\right) \pi\left(z_{n}\right) \\
=q^{2} \pi\left(z_{n}\right) \pi\left(z_{n+1}\right) \pi\left(z_{n+1}\right)^{*}+q^{2}\left(1-q^{2}\right) \sum_{k>n+1} \pi\left(z_{n}\right) \pi\left(z_{k}\right) \pi\left(z_{k}^{*}\right) \\
\quad-q^{2} \pi\left(z_{n+1}\right) \pi\left(z_{n}\right) \pi\left(z_{n+1}\right)^{*}-q^{2}\left(1-q^{2}\right) \sum_{k>n+1} \pi\left(z_{n}\right) \pi\left(z_{k}\right) \pi\left(z_{k}^{*}\right) \\
=q^{2}\left[\pi\left(z_{n}\right), \pi\left(z_{n+1}\right)\right] \pi\left(z_{n+1}\right)^{*} .
\end{aligned}
$$

2.

$$
\begin{aligned}
& {\left[\pi\left(z_{n}\right), \pi\left(z_{n+1}\right)\right]^{*}\left[\pi\left(z_{n}\right), \pi\left(z_{n+1}\right)\right]^{m} u} \\
& =\pi\left(z_{n}^{*}\right) \pi\left(z_{n+1}\right)^{*}\left[\pi\left(z_{n}\right), \pi\left(z_{n+1}\right)\right]^{m} u+\pi\left(z_{n+1}^{*}\right) \pi\left(z_{n}^{*}\right)\left[\pi\left(z_{n}\right), \pi\left(z_{n+1}\right)\right]^{m} u \\
& =C \pi\left(z_{n+1}^{*}\right) \sum_{l=0}^{m-1} q^{4 l}\left[\pi\left(z_{n}\right), \pi\left(z_{n+1}\right)\right]^{m-1-l} \pi\left(z_{n+1}\right)\left[\pi\left(z_{n}\right), \pi\left(z_{n+1}\right)\right]^{l} u
\end{aligned}
$$

(by part 7 of Proposition 4.6)

$$
=C \sum_{l=0}^{m-1} q^{4 l}\left[\pi\left(z_{n}\right), \pi\left(z_{n+1}\right)\right]^{m-1-l} \pi\left(z_{n+1}^{*}\right) \pi\left(z_{n+1}\right)\left[\pi\left(z_{n}\right), \pi\left(z_{n+1}\right)\right]^{l} u
$$

(by (4.1))

$$
\begin{aligned}
= & C \sum_{l=0}^{m-1} q^{4 l}\left[\pi\left(z_{n}\right), \pi\left(z_{n+1}\right)\right]^{m-1-l}\left(\pi\left(z_{n+1}\right) \pi\left(z_{n+1}\right)^{*}\right. \\
& \left.+\left(1-q^{2}\right) \sum_{k>i} \pi\left(z_{k}\right) \pi\left(z_{k}\right)^{*}\right)\left[\pi\left(z_{n}\right), \pi\left(z_{n+1}\right)\right]^{l} u \\
= & C\left[\pi\left(z_{n}\right), \pi\left(z_{n+1}\right)\right]^{m-1} u .
\end{aligned}
$$

From part 9 of Proposition 4.6, it follows that $\left[\pi\left(z_{n}\right), \pi\left(z_{n+1}\right)\right]^{\alpha_{0}} u \neq 0$. Further we have $\operatorname{ker} \pi\left(z_{i}\right) \subset \operatorname{ker} \pi\left(z_{2 n}^{*}\right)=\{0\}$; hence $u_{\alpha_{2}, \cdots, \alpha_{2 n-1}, \alpha_{0}} \neq 0$ for all $\left(\alpha_{2}, \cdots \alpha_{2 n-1}, \alpha_{0}\right) \in \mathbb{N}^{2 n-1}$. Therefore we can define

$$
e_{\alpha_{2}, \cdots, \alpha_{2 n-1}, \alpha_{0}}=\frac{u_{\alpha_{2}, \cdots, \alpha_{2 n-1}, \alpha_{0}}}{\left\|u_{\alpha_{2}, \cdots, \alpha_{2 n-1}, \alpha_{0}}\right\|} .
$$

Proposition 4.7. Assume $\left\{e_{\alpha_{2}, \cdots, \alpha_{2 n-1}, \alpha_{0}}:\left(\sum_{l=2}^{2 n-1} \alpha_{l}\right)+2 \alpha_{0} \leq L\right\}$ form an orthonormal basis for $\mathcal{H}_{\leq L}=\mathcal{H}_{0} \oplus \mathcal{H}_{1} \oplus \cdots \oplus \mathcal{H}_{L}$. If $2(r+s)+1 \leq L$, then

$$
\left[\pi\left(z_{n}\right), \pi\left(z_{n+1}\right)\right]^{r} \pi\left(z_{n+1}\right)\left[\pi\left(z_{n}\right), \pi\left(z_{n+1}\right)\right]^{s} u=C \pi\left(z_{n+1}\right)\left[\pi\left(z_{n}\right), \pi\left(z_{n+1}\right)\right]^{r+s} u,
$$


where $C$ is a non-zero constant.

Proof: It is enough to prove the statement for $r=1$. The condition ensures that,

$$
\left[\pi\left(z_{n}\right), \pi\left(z_{n+1}\right)\right] \pi\left(z_{n+1}\right)\left[\pi\left(z_{n}\right), \pi\left(z_{n+1}\right)\right]^{s} u \in \mathcal{H}_{\leq L}
$$

Hence

$$
\begin{aligned}
& {\left[\pi\left(z_{n}\right), \pi\left(z_{n+1}\right)\right] \pi\left(z_{n+1}\right)\left[\pi\left(z_{n}\right), \pi\left(z_{n+1}\right)\right]^{s} u} \\
& =\sum_{\left(\alpha_{2} \cdots \alpha_{2 n-1}, \alpha_{0}\right):\left(\sum_{i=2}^{2 n-1} \alpha_{i}\right)+2 \alpha_{0} \leq L} C\left(\alpha_{2}, \cdots, \alpha_{0}\right) e_{\alpha_{2}, \cdots, \alpha_{0}},
\end{aligned}
$$

where

$$
C\left(\alpha_{2}, \cdots, \alpha_{2 n-1}, \alpha_{0}\right)=\left\langle\left[\pi\left(z_{n}\right), \pi\left(z_{n+1}\right)\right] \pi\left(z_{n+1}\right)\left[\pi\left(z_{n}\right), \pi\left(z_{n+1}\right)\right]^{s} u, e_{\alpha_{2}, \cdots, \alpha_{2 n-1}, \alpha_{0}}\right\rangle .
$$

We will show that $C\left(\alpha_{2}, \cdots, \alpha_{2 n-1}, \alpha_{0}\right)=0$ if $\alpha_{n+1}=1$ and $\alpha_{0}=s+1$.

Case $1: \alpha_{i} \neq 0$ for some $i>n+1$.

Applying part 4 of the Proposition 4.6, we get

$$
\pi\left(z_{i}\right)^{*}\left[\pi\left(z_{n}\right), \pi\left(z_{n+1}\right)\right] \pi\left(z_{n+1}\right)\left[\pi\left(z_{n}\right), \pi\left(z_{n+1}\right)\right]^{s} u=0 .
$$

This shows that if $\alpha_{i} \neq 0$ for any $i \in\{n+2, n+3, \cdots, 2 n-1\}$ then

$$
C\left(\alpha_{2}, \cdots, \alpha_{2 n-1}, \alpha_{0}\right)=0
$$

Case 2: $\alpha_{n+1} \geq 1$ and $\alpha_{i}=0$ for all $i>n+1$.

$$
\begin{aligned}
& \pi\left(z_{n+1}\right)^{*}\left[\pi\left(z_{n}\right), \pi\left(z_{n+1}\right)\right] \pi\left(z_{n+1}\right)\left[\pi\left(z_{n}\right), \pi\left(z_{n+1}\right)\right]^{s} u \\
& =q^{2}\left[\pi\left(z_{n}\right), \pi\left(z_{n+1}\right)\right] \pi\left(z_{n+1}^{*} z_{n+1}\right)\left[\pi\left(z_{n}\right), \pi\left(z_{n+1}\right)\right]^{s} u . \\
& =q^{2}\left[\pi\left(z_{n}\right), \pi\left(z_{n+1}\right)\right] \pi\left(z_{n+1} z_{n+1}^{*}\right)\left[\pi\left(z_{n}\right), \pi\left(z_{n+1}\right)\right]^{s} u \\
& \quad+\sum_{k>n+1} q^{2}\left(1-q^{2}\right)\left[\pi\left(z_{n}\right), \pi\left(z_{n+1}\right)\right] \pi\left(z_{k} z_{k}^{*}\right)\left[\pi\left(z_{n}\right), \pi\left(z_{n+1}\right)\right]^{s} u \\
& =q^{4 s+2}\left(1-q^{2}\right)\left[\pi\left(z_{n}\right), \pi\left(z_{n+1}\right)\right]^{s+1} u \\
& \quad\left(\text { since } u \in \bigcap_{i=1}^{2 n-1} \operatorname{ker} \pi\left(z_{i}^{*}\right)\right) .
\end{aligned}
$$

Now,

$$
\begin{aligned}
& \left\langle u_{\alpha_{2}, \cdots, \alpha_{n+1}, 0, \cdots, 0, \alpha_{0}},\left[\pi\left(z_{n}\right), \pi\left(z_{n+1}\right)\right] \pi\left(z_{n+1}\right)\left[\pi\left(z_{n}\right), \pi\left(z_{n+1}\right)\right]^{s} u\right\rangle \\
& =\left\langle u_{\alpha_{2}, \cdots, \alpha_{n+1}-1,0, \cdots, 0, \alpha_{0}}, \pi\left(z_{n+1}^{*}\right)\left[\pi\left(z_{n}\right), \pi\left(z_{n+1}\right)\right] \pi\left(z_{n+1}\left[\pi\left(z_{n}\right), \pi\left(z_{n+1}\right)\right]^{s} u\right\rangle .\right. \\
& =\left\langle u_{\alpha_{2}, \cdots, \alpha_{n+1}-1,0, \cdots, 0, \alpha_{0}}, q^{4 s+2}\left(1-q^{2}\right)\left[\pi\left(z_{n}\right), \pi\left(z_{n+1}\right)\right]^{s+1} u\right\rangle . \\
& \begin{cases}\neq 0 & \text { if } \alpha_{n+1}=1, \alpha_{0}=s+1, \alpha_{n-1}=\cdots \alpha_{1}=0, \\
=0 & \text { otherwise } .\end{cases}
\end{aligned}
$$


Case 3: $\alpha_{i}=0$ for all $i \geq n+1$.

By using the commutation relations, we have

$$
\begin{aligned}
& \pi\left(z_{n}\right)^{*} u_{\alpha_{2}, \cdots, \alpha_{n}, 0, \cdots, 0, \alpha_{0}} \\
& =\pi\left(z_{n}\right)^{\alpha_{n}} \pi\left(z_{n}\right)^{*} \pi\left(z_{n-1}\right)^{\alpha_{n-1}} \cdots,\left[\pi\left(z_{n}\right), \pi\left(z_{n+1}\right)\right]^{\alpha_{0}} u \\
& \quad+\left(1-q^{2 \alpha_{n}}\right) \sum_{k>n} \pi\left(z_{n}\right)^{\alpha_{n}-1} \pi\left(z_{k}\right) \pi\left(z_{k}\right)^{*} \pi\left(z_{n-1}\right)^{\alpha_{n-1}} \cdots,\left[\pi\left(z_{n}\right), \pi\left(z_{n+1}\right)\right]^{\alpha_{0}} u . \\
& =C \pi\left(z_{n}\right)^{\alpha_{n}} \pi\left(z_{n-1}\right)^{\alpha_{n-1}} \cdots, \pi\left(z_{2}\right)^{\alpha_{2}} \pi\left(z_{n}\right)^{*}\left[\pi\left(z_{n}\right), \pi\left(z_{n+1}\right)\right]^{\alpha_{0}} u \\
& \quad+\left(1-q^{2 \alpha_{n}}\right) \sum_{k>n} \pi\left(z_{n}\right)^{\alpha_{n}-1} \pi\left(z_{k}\right) \pi\left(z_{k}\right)^{*} \pi\left(z_{n-1}\right)^{\alpha_{n-1}} \cdots,\left[\pi\left(z_{n}\right), \pi\left(z_{n+1}\right)\right]^{\alpha_{0}} u .
\end{aligned}
$$

for some non-zero constant $C$.

From part 5 of the Proposition 4.6, it follows that the second term of the right hand side is $C u_{\alpha_{2}, \cdots, \alpha_{n-1}, \alpha_{n}-1,0, \cdots, 0, \alpha_{0}}$. Also, the first term of the right hand side is

$$
\begin{aligned}
C \pi\left(z_{n}\right)^{\alpha_{n}} & \cdots, \pi\left(z_{2}\right)^{\alpha_{2}}\left[\pi\left(z_{n}\right), \pi\left(z_{n+1}\right)\right]^{\alpha_{0}} \pi\left(z_{n}\right)^{*} \\
& +C \pi\left(z_{n}\right)^{\alpha_{n}} \cdots, \pi\left(z_{2}\right)^{\alpha_{2}}\left(\sum_{l=0}^{\alpha-1} q^{4 l}\left[\pi\left(z_{n}\right), \pi\left(z_{n+1}\right)\right]^{\alpha_{0}-1-l} \pi\left(z_{n+1}\right)\left[\pi\left(z_{n}\right), \pi\left(z_{n+1}\right)\right]^{l}\right) u .
\end{aligned}
$$

Hence,

$$
\begin{aligned}
& \pi\left(z_{n+1}\right)^{*} \pi\left(z_{n}\right)^{*} u_{\alpha_{2}, \cdots, \alpha_{n}, 0, \cdots, 0, \alpha_{0}} \\
= & C \pi\left(z_{n}\right)^{\alpha_{n}} \cdots \pi\left(z_{2}\right)^{\alpha_{2}} \pi\left(z_{n+1}^{*}\right)\left(\sum_{l=0}^{\alpha_{0}-1} q^{4 l}\left[\pi\left(z_{n}\right), \pi\left(z_{n+1}\right)\right]^{\alpha_{0}-1-l} \pi\left(z_{n+1}\right)\left[\pi\left(z_{n}\right), \pi\left(z_{n+1}\right)\right]^{l}\right) u \\
= & C \pi\left(z_{n}\right)^{\alpha_{n}} \cdots \pi\left(z_{2}\right)^{\alpha_{2}}\left(\sum_{l=0}^{\alpha_{0}-1} q^{4 l}\left[\pi\left(z_{n}\right), \pi\left(z_{n+1}\right)\right]^{\alpha_{0}-1-l} \pi\left(z_{n+1}^{*}\right) \pi\left(z_{n+1}\right)\left[\pi\left(z_{n}\right), \pi\left(z_{n+1}\right)\right]^{l}\right) u \\
= & C \pi\left(z_{n}\right)^{\alpha_{n}} \cdots \pi\left(z_{2}\right)^{\alpha_{2}}\left(\sum _ { l = 0 } ^ { \alpha _ { 0 } - 1 } q ^ { 4 l } [ \pi ( z _ { n } ) , \pi ( z _ { n + 1 } ) ] ^ { \alpha _ { 0 } - 1 - l } \left(\pi\left(z_{n+1}\right) \pi\left(z_{n+1}^{*}\right)\right.\right. \\
& \left.\left.+\sum_{k>n+1} \pi\left(z_{k}\right) \pi\left(z_{k}^{*}\right)\right)\left[\pi\left(z_{n}\right), \pi\left(z_{n+1}\right)\right]^{l}\right) u \\
= & C \pi\left(z_{n}\right)^{\alpha_{n}} \cdots \pi\left(z_{2}\right)^{\alpha_{2}}\left[\pi\left(z_{n}\right), \pi\left(z_{n+1}\right)\right]^{\alpha_{0}-1} u .
\end{aligned}
$$

By the above calculation and by Proposition 4.6, we have

$$
\begin{aligned}
& \left\langle u_{\alpha_{2}, \cdots, \alpha_{n}, 0 \cdots, 0, \alpha_{0}},\left[\pi\left(z_{n}\right), \pi\left(z_{n+1}\right)\right] \pi\left(z_{n+1}\right)\left[\pi\left(z_{n}\right), \pi\left(z_{n+1}\right)\right]^{s} u\right\rangle \\
& =\left\langle\left[\pi\left(z_{n}\right), \pi\left(z_{n+1}\right)\right]^{*} u_{\alpha_{2}, \cdots, \alpha_{n}, 0 \cdots, 0, \alpha_{0}}, \pi\left(z_{n+1}\right)\left[\pi\left(z_{n}\right), \pi\left(z_{n+1}\right)\right]^{s} u\right\rangle \\
& =\left\langle\pi\left(z_{n+1}^{*} z_{n}^{*}-z_{n}^{*} z_{n+1}^{*}\right) u_{\alpha_{2}, \cdots, \alpha_{n}, 0 \cdots, 0, \alpha_{0}}, \pi\left(z_{n+1}\right)\left[\pi\left(z_{n}\right), \pi\left(z_{n+1}\right)\right]^{s} u\right\rangle \\
& =\left\langle\pi\left(z_{n+1}^{*} z_{n}^{*}\right) u_{\alpha_{2}, \cdots, \alpha_{n}, 0 \cdots, 0, \alpha_{0}}, \pi\left(z_{n+1}\right)\left[\pi\left(z_{n}\right), \pi\left(z_{n+1}\right)\right]^{s} u\right\rangle \\
& =\left\langle C \pi\left(z_{n}\right)^{\alpha_{n}} \pi\left(z_{n-1}\right)^{\alpha_{n-1}} \cdots, \pi\left(z_{2}\right)^{\alpha_{2}}\left[\pi\left(z_{n}\right), \pi\left(z_{n+1}\right)\right]^{\alpha_{0}-1} u, \pi\left(z_{n+1}\right)\left[\pi\left(z_{n}\right), \pi\left(z_{n+1}\right)\right]^{s} u\right\rangle \\
& =\left\langle C \pi\left(z_{n+1}^{*}\right) \pi\left(z_{n}\right)^{\alpha_{n}} \pi\left(z_{n-1}\right)^{\alpha_{n-1}} \cdots, \pi\left(z_{2}\right)^{\alpha_{2}}\left[\pi\left(z_{n}\right), \pi\left(z_{n+1}\right)\right]^{\alpha_{0}-1} u,\left[\pi\left(z_{n}\right), \pi\left(z_{n+1}\right)\right]^{s} u\right\rangle \\
& =0
\end{aligned}
$$


This proves the claim.

Lemma 4.8. Let $\pi$ be an irreducible representation on a Hilbert space $\mathcal{H}$ with $\pi\left(z_{2 n}\right) \neq 0$. Then $\left\{e_{\alpha_{2}, \alpha_{2}, \cdots, \alpha_{2 n-1}, \alpha_{0}}:\left(\alpha_{2}, \alpha_{3}, \cdots \alpha_{2 n-1}, \alpha_{0}\right) \in \mathbb{N}^{2 n-1}\right\}$ defined in equation (4.12), form an orthonormal basis for $\mathcal{H}$.

Proof: From Corollary 4.5, it is enough to show that for $\alpha \neq \beta, u_{\alpha}$ is orthogonal to $u_{\beta}$. We apply induction on $L_{\alpha}:=\left(\sum_{i=2}^{2 n-1} \alpha_{i}\right)+2 \alpha_{0}$. For $L_{\alpha}=0$, claim is true as $u \neq 0$. Assume the hypothesis for $L_{\alpha} \leq N-1$. Note that $u_{\alpha_{2}, \cdots, \alpha_{2 n-1}, \alpha_{0}} \in \mathcal{H}_{L_{\alpha}}$. Hence, by induction hypothesis and Corollary 4.5 , it follows that $\left\{e_{\alpha_{2}, \cdots, \alpha_{2 n-1}, \alpha_{0}}:\left(\sum_{l=2}^{2 n-1} \alpha_{l}\right)+2 \alpha_{0}=m\right\}$ form an orthonormal basis of $\mathcal{H}_{m}$ for $m \leq N-1$.

If $\alpha$ and $\beta$ are such that $L_{\alpha} \neq L_{\beta}$, then $u_{\alpha} \in \mathcal{H}_{L_{\alpha}}$ and $u_{\beta} \notin \mathcal{H}_{L_{\alpha}}$, which shows that $u_{\alpha}$ and $u_{\beta}$ are orthogonal. Take $\alpha$ and $\beta$ such that $L_{\alpha}=L_{\beta}=N$. Assume $\alpha_{i} \neq 0$ for some $i>n$. Choose maximum such $i$. From part 6 of Proposition 4.6, it follows that

$$
\begin{aligned}
\left\langle u_{\alpha}, u_{\beta}\right\rangle & =\left\langle u_{\alpha_{2}, \cdots, \alpha_{i-1}, \alpha_{i}-1,0, \cdots, 0, \alpha_{0}}, \pi\left(z_{i}^{*}\right) u_{\beta}\right\rangle \\
& =\left\langle u_{\alpha_{2}, \cdots, \alpha_{i-1}, \alpha_{i}-1,0, \cdots, 0, \alpha_{0}}, C u_{\beta_{2}, \cdots, \beta_{i-1}, \beta_{i}-1, \beta_{i+1}, \cdots, \beta_{2 n-1}, \beta_{0}}\right\rangle
\end{aligned}
$$

where $C$ is a non zero constant. Now, by using induction we get $\left\langle u_{\alpha}, u_{\beta}\right\rangle \neq 0$ if and only if $\alpha=\beta$. Hence it is enough to consider $\alpha$ and $\beta$ such that $\alpha_{i}=\beta_{i}=0$ for $i>n$. Let $\alpha_{n} \neq 0$.

$$
\begin{aligned}
& \pi\left(z_{n}^{*}\right) u_{\beta_{2}, \cdots, \beta_{n}, 0, \cdots, 0, \beta_{0}} \\
& =\left(\pi\left(z_{n}^{\beta_{n}}\right) \pi\left(z_{n}^{*}\right)+\sum_{k>i} C \pi\left(z_{n}^{\beta_{n}-1}\right) \pi\left(z_{k}\right) \pi\left(z_{k}^{*}\right)\right) u_{\beta_{2}, \cdots, \beta_{n-1}, 0, \cdots, 0, \beta_{0}} \\
& =C \pi\left(z_{n}^{\beta_{n}}\right) \pi\left(z_{n+1}\right) u_{\beta_{2}, \cdots, \beta_{n-1}, 0, \cdots, 0, \beta_{0}-1}+C u_{\beta_{2}, \cdots, \beta_{n}-1,0, \cdots, 0, \beta_{0}}
\end{aligned}
$$

(by Proposition 4.7)

$$
\begin{aligned}
& =\left(C \pi\left(z_{n+1}\right) \pi\left(z_{n}^{\beta_{n}}\right)+\sum_{k>n+1} C \pi\left(z_{k}\right) \pi\left(z_{k}^{\prime}\right) \pi\left(z_{n}^{\beta_{n}-1}\right)\right) u_{\beta_{2}, \cdots, \beta_{n-1}, 0, \cdots, 0, \beta_{0}-1} \\
& \quad+C u_{\beta_{2}, \cdots, \beta_{n}-1,0, \cdots, 0, \beta_{0} .}
\end{aligned}
$$

Hence

$$
\begin{aligned}
& \left\langle u_{\alpha}, u_{\beta}\right\rangle \\
& =\left\langle u_{\alpha_{2}, \cdots, \alpha_{n-1}, \alpha_{n}-1,0, \cdots, 0, \alpha_{0}}, \pi\left(z_{n}^{*}\right) u_{\beta}\right\rangle \\
& =\left\langle u_{\alpha_{2}, \cdots, \alpha_{n-1}, \alpha_{n}-1,0, \cdots, 0, \alpha_{0}}, C u_{\beta_{2}, \cdots, \beta_{n}-1,0, \cdots, 0, \beta_{0}}+C \pi\left(z_{2 n}\right) \pi\left(z_{1}\right) u_{\beta_{2}, \cdots, \beta_{n}-1,0, \cdots, 0, \beta_{0}-1}\right\rangle \\
& =\left\langle u_{\alpha_{2}, \cdots, \alpha_{n-1}, \alpha_{n}-1,0, \cdots, 0, \alpha_{0}}, C u_{\beta_{2}, \cdots, \beta_{n}-1,0, \cdots, 0, \beta_{0}}\right\rangle \\
& \quad \quad \quad\left\langle\pi\left(z_{1}^{*}\right) u_{\alpha_{2}, \cdots, \alpha_{n-1}, \alpha_{n}-1,0, \cdots, 0, \alpha_{0}}, C u_{\beta_{2}, \cdots, \beta_{n}-1,0, \cdots, 0, \beta_{0}-1}\right\rangle \\
& =\left\langle u_{\alpha_{2}, \cdots, \alpha_{n-1}, \alpha_{n}-1,0, \cdots, 0, \alpha_{0}}, C u_{\beta_{2}, \cdots, \beta_{n}-1,0, \cdots, 0, \beta_{0}}\right\rangle \\
& \quad+\left\langle u_{\alpha_{2}, \cdots, \alpha_{n-1}, \alpha_{n}-1,0, \cdots, 0, \alpha_{0}-1}, C u_{\beta_{2}, \cdots, \beta_{n}-1,0, \cdots, 0, \beta_{0}-1}\right\rangle .
\end{aligned}
$$


Again induction proves the claim. So, we will consider $\alpha$ and $\beta$ such that $\alpha_{i}=\beta_{i}=0$ for $i \geq n$. Assume that for some $i \in\{2,3, \cdots, n-1\}, \alpha_{i} \neq 0$ or $\beta_{i} \neq 0$. Choose maximum such $i$. Without loss of generality, we assume that $\alpha_{i} \neq 0$.

$$
\begin{aligned}
\pi\left(z_{i}^{*}\right) u_{\beta_{2}, \cdots, \beta_{i}, 0, \cdots, 0, \beta_{0}} \\
=\left(\pi\left(z_{i}\right)^{\beta_{i}} \pi\left(z_{i}\right)^{*}+q^{2 \rho_{i}}\left(1-q^{2 \beta_{i}}\right) \pi\left(z_{i}\right)^{\beta_{i}-1} \pi\left(z_{i^{\prime}}\right) \pi\left(z_{i^{\prime}}\right)^{*}\right. \\
\left.\quad+\left(1-q^{2 \beta_{i}}\right) \sum_{k>i} \pi\left(z_{i}\right)^{\beta_{i}-1} \pi\left(z_{k}\right) \pi\left(z_{k}\right)^{*}\right) \pi\left(z_{i-1}\right)^{\beta_{i-1}} \cdots \pi\left(z_{2}\right)^{\beta_{2}}\left[\pi\left(z_{n}\right), \pi\left(z_{n+1}\right)\right]^{\beta_{0}} u \\
=C \pi\left(z_{i}\right)^{\beta_{i}} \pi\left(z_{i}^{\prime}\right) \pi\left(z_{i-1}\right)^{\beta_{i-1}} \cdots \pi\left(z_{2}\right)^{\beta_{2}}\left[\pi\left(z_{n}\right), \pi\left(z_{n+1}\right)\right]^{\beta_{0}-1} u+C u_{\beta_{2}, \cdots, \beta_{i}-1,0, \cdots, 0, \beta_{0}} \\
\left.\quad+\sum_{i<k \leq n} C \pi\left(z_{i}\right)^{\beta_{i}-1} \pi\left(z_{k}\right) \pi\left(z_{k}\right)^{*}\right) \pi\left(z_{i-1}\right)^{\beta_{i-1}} \cdots, \pi\left(z_{2}\right)^{\beta_{2}}\left[\pi\left(z_{n}\right), \pi\left(z_{n+1}\right)\right]^{\beta_{0}-1} u . \\
=C \pi\left(z_{i}^{\prime}\right) \pi\left(z_{i}\right)^{\beta_{i}} \pi\left(z_{i-1}\right)^{\beta_{i-1}} \cdots \pi\left(z_{2}\right)^{\beta_{2}}\left[\pi\left(z_{n}\right), \pi\left(z_{n+1}\right)\right]^{\beta_{0}-1} u+C u_{\beta_{2}, \cdots, \beta_{i}-1,0, \cdots, 0, \beta_{0}} \\
\left.\quad+\sum_{k>i^{\prime}} C \pi\left(z_{k}\right)\right) \pi\left(z_{k^{\prime}}\right) \pi\left(z_{i}\right)^{\beta_{i}-1} \pi\left(z_{i-1}\right)^{\beta_{i-1}} \cdots, \pi\left(z_{2}\right)^{\beta_{2}}\left[\pi\left(z_{n}\right), \pi\left(z_{n+1}\right)\right]^{\beta_{0}-1} u \\
\left.\quad+\sum_{i<k \leq n} C \pi\left(z_{i}\right)^{\beta_{i}-1} \pi\left(z_{k^{\prime}}\right)\right) \pi\left(z_{k}\right) \pi\left(z_{i-1}\right)^{\beta_{i-1}} \cdots, \pi\left(z_{2}\right)^{\beta_{2}}\left[\pi\left(z_{n}\right), \pi\left(z_{n+1}\right)\right]^{\beta_{0}-1} u \\
=C u_{\beta_{2}, \cdots, \beta_{i}-1,0, \cdots, 0, \beta_{0}} \\
\left.\quad+\sum_{n<k \leq 2 n} C \pi\left(z_{k}\right)\right) \pi\left(z_{k^{\prime}}\right) \pi\left(z_{i}\right)^{\beta_{i}-1} \pi\left(z_{i-1}\right)^{\beta_{i-1} \cdots, \pi\left(z_{2}\right)^{\beta_{2}}\left[\pi\left(z_{n}\right), \pi\left(z_{n+1}\right)\right]^{\beta_{0}-1} u .}
\end{aligned}
$$

Hence

$$
\begin{aligned}
& \left\langle u_{\alpha}, u_{\beta}\right\rangle \\
& =\left\langle u_{\alpha_{2}, \cdots, \alpha_{i-1}, \alpha_{i}-1,0, \cdots, 0, \alpha_{0}}, \pi\left(z_{i}^{*}\right) u_{\beta_{2}, \cdots, \beta_{i}, 0, \cdots, 0, \beta_{0}}\right\rangle \\
& =\left\langle u_{\alpha_{2}, \cdots, \alpha_{i-1}, \alpha_{i}-1,0, \cdots, 0, \alpha_{0}}, C u_{\beta_{2}, \cdots, \beta_{i}-1,0, \cdots, 0, \beta_{0}}+C \pi\left(z_{2 n}\right) \pi\left(z_{1}\right) u_{\beta_{2}, \cdots, \beta_{i}-1,0, \cdots, 0, \beta_{0}-1}\right\rangle \\
& =\left\langle u_{\alpha_{2}, \cdots, \alpha_{i-1}, \alpha_{i}-1,0, \cdots, 0, \alpha_{0}}, C u_{\beta_{2}, \cdots, \beta_{i}-1,0, \cdots, 0, \beta_{0}}\right\rangle \\
& \quad \quad \quad\left\langle\pi\left(z_{1}^{*}\right) u_{\alpha_{2}, \cdots, \alpha_{i-1}, \alpha_{i}-1,0, \cdots, 0, \alpha_{0}}, C u_{\beta_{2}, \cdots, \beta_{i}-1,0, \cdots, 0, \beta_{0}-1}\right\rangle \\
& =\left\langle u_{\alpha_{2}, \cdots, \alpha_{i-1}, \alpha_{i}-1,0, \cdots, 0, \alpha_{0}}, C u_{\beta_{2}, \cdots, \beta_{i}-1,0, \cdots, 0, \beta_{0}}\right\rangle \\
& \quad+\left\langle u_{\alpha_{2}, \cdots, \alpha_{n-1}, \alpha_{i}-1,0, \cdots, 0, \alpha_{0}-1}, C u_{\beta_{2}, \cdots, \beta_{i}-1,0, \cdots, 0, \beta_{0}-1}\right\rangle .
\end{aligned}
$$

Again induction will settle the claim. Now we take $\alpha$ and $\beta$ such that $\alpha_{i}=\beta_{i}=0$ for all $i \neq 0$. Then from part 9 of Proposition 4.6, it follows that

$$
\left[\pi\left(z_{n}\right), \pi\left(z_{n+1}\right)\right]^{*}\left[\pi\left(z_{n}\right), \pi\left(z_{n+1}\right)\right]^{\beta_{0}} u=C\left[\pi\left(z_{n}\right), \pi\left(z_{n+1}\right)\right]^{\beta_{0}-1} .
$$

Hence

$$
\begin{aligned}
\left\langle u_{\alpha}, u_{\beta}\right\rangle & =\left\langle\left[\pi\left(z_{n}\right), \pi\left(z_{n+1}\right)\right]^{\alpha_{0}} u,\left[\pi\left(z_{n}\right), \pi\left(z_{n+1}\right)\right]^{\beta_{0}} u\right\rangle \\
& =\left\langle\left[\pi\left(z_{n}\right), \pi\left(z_{n+1}\right)\right]^{\alpha_{0}-1} u,\left[\pi\left(z_{n}\right), \pi\left(z_{n+1}\right)\right]^{*}\left[\pi\left(z_{n}\right), \pi\left(z_{n+1}\right)\right]^{\beta_{0}} u\right\rangle \\
& =\left\langle\left[\pi\left(z_{n}\right), \pi\left(z_{n+1}\right)\right]^{\alpha_{0}-1} u, C\left[\pi\left(z_{n}\right), \pi\left(z_{n+1}\right)\right]^{\beta_{0}-1} u\right\rangle
\end{aligned}
$$


This completes the proof.

Corollary 4.9. If $\pi$ and $\pi^{\prime}$ are two irreducible representations of $C\left(H_{q}^{2 n}\right)$ with $\pi\left(z_{2 n}\right) \neq$ 0 and $\pi^{\prime}\left(z_{2 n}\right) \neq 0$, then for all $\alpha \in \mathbb{N}^{2 n-1}$, we have

$$
\left\|u_{\alpha}\right\|=\left\|u_{\alpha}^{\prime}\right\| .
$$

where $u_{\alpha}$ and $u_{\alpha}^{\prime}$ are defined as above.

Now we aim to find all irreducible representations of $C\left(H_{q}^{2 n}\right)$. One way is to do explicit calculations to determine the operators $z_{1}, z_{2}, \cdots, z_{2 n}$ as done in case of the odd dimensional quantum spheres. But in this case, computations are more complicated. So, to avoid complicated calculations, we show that one can completely determine an irreducible representation $\pi$ of $C\left(H_{q}^{2 n}\right)$ given that $\pi(\omega) \neq 0$ and $\pi\left(z_{2 n}\right) u=t u$ for some fixed $t \in \mathbb{T}$. Then we use representation of the quantum Stiefel manifold $C\left(S P_{q}(2 n) / S P_{q}(2 n-2)\right)$ to get explicit description of the representation.

Theorem 4.10. Let $\pi$ and $\pi^{\prime}$ be irreducible representations of $C\left(H_{q}^{2 n}\right)$ on a Hilbert space $\mathcal{H}$ and $\mathcal{H}^{\prime}$ respectively such that $\left.\pi\left(z_{2 n}\right)\right|_{\bigcap_{i=1}^{2 n-1} \operatorname{ker} \pi\left(z_{i}^{*}\right)}=t I=\left.\pi^{\prime}\left(z_{2 n}\right)\right|_{\bigcap_{i=1}^{2 n-1} \operatorname{ker} \pi^{\prime}\left(z_{i}^{*}\right)}$ for $t \in \mathbb{T}$. Then $\pi$ and $\pi^{\prime}$ are equivalent.

Proof: Without loss of generality, we can take $t=1$. Let $u$ and $u^{\prime}$ are unit vectors in $\bigcap_{i=1}^{2 n-1} \operatorname{ker} \pi\left(z_{i}^{*}\right)$ and $\bigcap_{i=1}^{2 n-1} \operatorname{ker} \pi^{\prime}\left(z_{i}^{*}\right)$ respectively. From Lemma 4.8, we have canonical orthonormal bases for $\mathcal{H}$ and $\mathcal{H}^{\prime}$ given by

$$
\left\{e_{\alpha_{2}, \alpha_{3}, \cdots, \alpha_{2 n-1}, \alpha_{0}}:\left(\alpha_{2}, \alpha_{3}, \cdots \alpha_{2 n-1}, \alpha_{0}\right) \in \mathbb{N}^{2 n-1}\right\}
$$

and

$$
\left\{e_{\alpha_{2}, \alpha_{3}, \cdots, \alpha_{2 n-1}, \alpha_{0}}^{\prime}:\left(\alpha_{2}, \alpha_{3}, \cdots \alpha_{2 n-1}, \alpha_{0}\right) \in \mathbb{N}^{2 n-1}\right\}
$$

respectively. Define $U: \mathcal{H} \rightarrow \mathcal{H}^{\prime}$ by

$$
U: e_{\alpha_{2}, \alpha_{3}, \cdots, \alpha_{2 n-1}, \alpha_{0}} \longmapsto e_{\alpha_{2}, \alpha_{3}, \cdots, \alpha_{2 n-1}, \alpha_{0}}^{\prime}
$$

From Corollary [4.9, $U\left(u_{\alpha_{2}, \alpha_{3}, \cdots, \alpha_{2 n-1}, \alpha_{0}}\right)=u_{\alpha_{2}, \alpha_{3}, \cdots, \alpha_{2 n-1}, \alpha_{0}}^{\prime}$. We know, $\mathcal{H}=\oplus_{m \in \mathbb{N}} \mathcal{H}_{m}$ and $\mathcal{H}^{\prime}=\oplus_{m \in \mathbb{N}} \mathcal{H}_{m}^{\prime}$. where $\mathcal{H}_{m}$ and $\mathcal{H}_{m}^{\prime}$ are the eigenspaces of $\pi(\omega)$ and $\pi^{\prime}(\omega)$ respectively, corresponding to the eigenvalue $q^{2 m}$. Clearly $U\left(\mathcal{H}_{m}\right)=\mathcal{H}_{m}^{\prime}$. We need to show that, $U \pi\left(z_{i}\right) U^{*}=$ $\pi^{\prime}\left(z_{i}\right)$, or equivalently $U \pi\left(z_{i}^{*}\right) U^{*}=\pi^{\prime}\left(z_{i}^{*}\right)$ for all $i \in\{1,2, \cdots, 2 n\}$. We split the proof into several parts.

(A) $i=2 n$ :

$$
\begin{aligned}
& \pi\left(z_{2 n}^{*}\right) u_{\alpha_{2}, \cdots, \alpha_{2 n-1}, \alpha_{0}}=q^{\left(\sum_{l=2}^{2 n-1} \alpha_{l}\right)+2 \alpha_{0}} u_{\alpha_{2}, \cdots, \alpha_{2 n-1}, \alpha_{0}} . \\
& \pi^{\prime}\left(z_{2 n}^{*}\right) u_{\alpha_{2}, \cdots, \alpha_{2 n-1}, \alpha_{0}}=q^{\left(\sum_{l=2}^{2 n-1} \alpha_{l}\right)+2 \alpha_{0}} u_{\alpha_{2}, \cdots, \alpha_{2 n-1}, \alpha_{0}}^{\prime}
\end{aligned}
$$


(B) $n<i<2 n$ :

$$
\begin{aligned}
\pi\left(z_{i}^{*}\right) u_{\alpha_{2}, \cdots, \alpha_{2 n-1}, \alpha_{0}} & =C u_{\alpha_{2}, \cdots, \alpha_{i-1}, \alpha_{i}, \cdots, \alpha_{2 n-1}, \alpha_{0}} \\
\pi^{\prime}\left(z_{i}^{*}\right) u_{\alpha_{2}, \cdots, \alpha_{2 n-1}, \alpha_{0}}^{\prime} & =C u_{\alpha_{2}, \cdots, \alpha_{i-1}, \alpha_{i}, \cdots, \alpha_{2 n-1}, \alpha_{0}}^{\prime} .
\end{aligned}
$$

Note that the constant $C$ is same in both equations. Hence we have,

$$
U \pi\left(z_{i}^{*}\right) U^{*}=\pi^{\prime}\left(z_{i}\right) \quad \text { for } n<i \leq 2 n .
$$

(C) $i=n$ :

In this case, we will use induction on the dimension of the eigenspaces of $\pi(\omega)$. For $m=0$, we have $\pi\left(z_{i}^{*}\right) u=0=\pi^{\prime}\left(z_{i}^{*}\right) u^{\prime}$. Assume that $U \pi\left(z_{i}\right) U_{\mid \mathcal{H}_{\leq m}}^{*}=\pi^{\prime}\left(z_{i}\right) \mathcal{H}_{\leq m}^{\prime}$. Take $u_{\alpha_{2}, \cdots, \alpha_{2 n-1}, \alpha_{0}} \in$ $\mathcal{H}_{m+1}$.

Case $1: \alpha_{j} \neq 0$ for some $j>n$, and $\alpha_{k}=0$ for all $k>j$.

$$
\begin{aligned}
& \pi\left(z_{n}^{*}\right) u_{\alpha_{2}, \cdots, \alpha_{j}, 0, \cdots, 0, \alpha_{0}}= C \pi\left(z_{j}\right) \pi\left(z_{n}^{*}\right) u_{\alpha_{2}, \cdots, \alpha_{j}-1,0, \cdots, 0, \alpha_{0},}, \quad \quad \text { by (4.1) } \\
&= C \pi\left(z_{j}\right) U^{*} \pi^{\prime}\left(z_{n}^{*}\right) u_{\alpha_{2}, \cdots, \alpha_{j}-1,0, \cdots, 0, \alpha_{0}}^{\prime}, \\
& \quad \quad \quad \text { by induction) } \\
&=C U^{*} \pi^{\prime}\left(z_{j}\right) U U^{*} \pi^{\prime}\left(z_{n}^{*}\right) u_{\alpha_{2}, \cdots, \alpha_{j}-1,0, \cdots, 0, \alpha_{0}}^{\prime}, \\
&=C U^{*} \pi^{\prime}\left(z_{j}\right) \pi^{\prime}\left(z_{n}^{*}\right) u_{\alpha_{2}, \cdots, \alpha_{j}-1,0, \cdots, 0, \alpha_{0}}^{\prime}, \\
&=\pi^{\prime}\left(z_{n}\right) u_{\alpha_{2}, \cdots, \alpha_{j}, 0, \cdots, 0, \alpha_{0} .}^{\prime}
\end{aligned}
$$

Case $2: \alpha_{j}=0$ for all $j>n$.

From part 7, part 8 of the Proposition 4.6 and Proposition 4.7, we have

$$
\begin{aligned}
\pi\left(z_{n}^{*}\right) u_{\alpha_{2}, \cdots, \alpha_{n}, 0, \cdots, 0, \alpha_{0}} & =C u_{\alpha_{2}, \cdots, \alpha_{n}-1,0, \cdots, 0, \alpha_{0}}+C u_{\alpha_{2}, \cdots, \alpha_{n}, 1,0, \cdots, 0, \alpha_{0}-1} . \\
\pi^{\prime}\left(z_{n}^{*}\right) u_{\alpha_{1}, \cdots, \alpha_{n}, 0, \cdots, 0, \alpha_{0}}^{\prime} & =C u_{\left(\alpha_{2}, \cdots, \alpha_{n}-1,0, \cdots, 0, \alpha_{0}\right.}^{\prime}+C u_{\left(\alpha_{2}, \cdots, \alpha_{n}, 1,0, \cdots, 0, \alpha_{0}-1\right)}^{\prime} .
\end{aligned}
$$

Hence we get

$$
U \pi\left(z_{n}\right)^{*} U^{*}=\pi^{\prime}\left(z_{n}\right)
$$

(D) $1<i<n$ :

Case $1: \alpha_{j} \neq 0$ for some $j>i$, and $\alpha_{k}=0$ for all $k>j$.

This follows exactly as in the case $i=n$.

Case $2: \alpha_{j}=0$, for all $j \geq i$.

It follows from part 2 of the Proposition [4.6 and by using the fact $U \pi\left(z_{k}\right)^{*} U^{*}=\pi^{\prime}\left(z_{k}\right)$ for all $k>i$. 
Case $3: \alpha_{j}=0$, for all $j \geq i$.

From part 8 and part 11 of the Proposition 4.6, we have

$$
\begin{aligned}
\pi\left(z_{i}\right)^{*} u_{\alpha_{2}, \cdots, \alpha_{i-1}, 0, \cdots, 0, \alpha_{0}} & =C u_{\alpha_{2}, \cdots, \alpha_{i-1}, 0, \cdots, 0,} \underbrace{}_{i^{\prime}-1} \underbrace{1}_{\text {th place }}, 0, \cdots, 0, \alpha_{0} \\
\pi^{\prime}\left(z_{i}\right)^{*} u_{\alpha_{2}, \cdots, \alpha_{i-1}, 0, \cdots, 0, \alpha_{0}} & =C u_{\alpha_{2}, \cdots, \alpha_{i-1}, 0, \cdots, 0,}^{\prime} \underbrace{}_{i^{\prime}-1} \underbrace{1}_{\text {th place }}, 0, \cdots, 0, \alpha_{0}
\end{aligned}
$$

which settles the claim for $1<i<n$.

(E) For $i=1$ :

We again use induction. Take $u_{\alpha}$ such that $\alpha_{j} \neq 0$ for some $j \neq 0$. Choose $j$ to be $\max$ $\left\{k: \alpha_{k} \neq 0\right\}$.

$$
\begin{aligned}
\pi\left(z_{1}\right)^{*} u_{\alpha_{2} \cdots, \alpha_{j}, 0, \cdots, 0, \alpha_{0}} & =C \pi\left(z_{j}\right) \pi\left(z_{1}\right)^{*} u_{\alpha_{2}, \cdots, \alpha_{j}-1,0, \cdots, 0, \alpha_{0}} \\
& =C U^{*} \pi^{\prime}\left(z_{j}\right) U U^{*} \pi^{\prime}\left(z_{1}\right)^{*} u_{\alpha_{2}, \cdots, \alpha_{j}-1,0, \cdots, 0, \alpha_{0}}^{\prime} \\
& =C U^{*} \pi^{\prime}\left(z_{j}\right) \pi^{\prime}\left(z_{1}\right)^{*} u_{\alpha_{2}, \cdots, \alpha_{j}-1,0, \cdots, 0, \alpha_{0}}^{\prime} \\
& =\pi^{\prime}\left(z_{1}\right)^{*} u_{\alpha_{2} \cdots, \alpha_{j}, 0, \cdots, 0, \alpha_{0}}^{\prime}
\end{aligned}
$$

For $\alpha$ such that $\alpha_{j}=0$, for all $j \neq 0$, it follows from part 12 of the Proposition 4.6 and induction. Hence, we have $U \pi\left(z_{i}^{*}\right) U^{*}=\pi^{\prime}\left(z_{i}^{*}\right)$ for $1 \leq i \leq 2 n$, which proves the claim.

We will now discuss the general case. Let $\pi$ be an irreducible representation of $C\left(H_{q}^{2 n}\right)$ on a Hilbert space $\mathcal{H}$ such that $\pi\left(z_{2 n}\right)=\pi\left(z_{2 n-1}\right)=\cdots=\pi\left(z_{k+1}\right)=0$, and $\pi\left(z_{k}\right) \neq 0$. It follows from (4.8) that $z_{k}$ is normal. Denote $z_{k}^{*} z_{k}$ by $\omega$. By the same reasoning, $\mathcal{H}$ decomposes as

$$
\mathcal{H}=\oplus_{m \in \mathbb{N}} \mathcal{H}_{m}
$$

where $\mathcal{H}_{m}$ is the eigenspace of $\pi(\omega)$ corresponding to the eigenvalue $q^{2 m}$.

Let $K$ be a subspace of $\bigcap_{i=1}^{k-1} \operatorname{ker} \pi\left(z_{i}^{*}\right)$ invariant under the $C^{*}$-algebra generated by $\pi\left(z_{k}\right)$. Define

$$
\mathcal{H}^{K}=\text { linear span }\left\{\pi\left(z_{1}\right)^{\alpha_{1}} \pi\left(z_{2}\right)^{\alpha_{2}} \cdots \pi\left(z_{k-1}\right)^{\alpha_{k-1}} h: h \in K\right\} .
$$

In the same way, one can show that, $\mathcal{H}^{K}$ is an invariant subspace of a representation $\pi$ and hence by irreducibility of the representation, $\bigcap_{i=1}^{k-1} \operatorname{ker} \pi\left(z_{i}^{*}\right)$ is one dimensional. Pick $u \in$ $\bigcap_{i=1}^{k-1}$ ker $\pi\left(z_{i}^{*}\right)=\mathcal{H}_{0}$. As $\pi\left(z_{k}\right)$ keeps $\mathcal{H}_{0}$ invariant and $\pi\left(z_{k}\right)_{\mid \mathcal{H}_{0}}$ is a unitary operator, we get $\pi\left(z_{k}\right) u=t u$ for some $t \in \mathbb{T}$.

Theorem 4.11. Let $1 \leq k \leq 2 n$ and let $\pi$ be an irreducible representation of $C\left(H_{q}^{2 n}\right)$ on a Hilbert space $\mathcal{H}$ such that $\pi\left(z_{2 n}\right)=\pi\left(z_{2 n-1}\right)=\cdots=\pi\left(z_{k+1}\right)=0$. Assume $\pi\left(z_{k}\right) u=t u, t \in \mathbb{T}$, 
where $u$ is defined as above. Then $\pi$ is the unique representation up to equivalence which satisfies these conditions.

Proof: This is essentially the previous proof with some minor modifications.

Case $1: k>n$.

Define

$$
\begin{aligned}
& u_{\alpha_{1}, \alpha_{2}, \cdots, \alpha_{2 n-k}, \alpha_{2 n-k+2}, \cdots, \alpha_{k-1}, \alpha_{0}} \\
& =\pi\left(z_{1}\right)^{\alpha_{1}} \cdots \pi\left(z_{2 n-k}\right)^{\alpha_{2 n-k}} \pi\left(z_{k-1}\right)^{\alpha_{k-1}} \cdots \pi\left(z_{2 n-k+2}\right)^{\alpha_{2 n-k+2}}\left[\pi\left(z_{n}\right), \pi\left(z_{n+1}\right)\right]^{\alpha_{0}} .
\end{aligned}
$$

where $\alpha_{i} \in \mathbb{N}$. (Note the differences between the definition of $u_{\alpha}$ given above and that given in equation (4.11).

$$
e_{\alpha_{1}, \alpha_{2}, \cdots, \alpha_{2 n-k}, \alpha_{2 n-k+2}, \cdots, \alpha_{k-1}, \alpha_{0}}=\frac{u_{\alpha_{1}, \alpha_{2}, \cdots, \alpha_{2 n-k}, \alpha_{2 n-k+2}, \cdots, \alpha_{k-1}, \alpha_{0}}}{\left\|u_{\alpha_{1}, \alpha_{2}, \cdots, \alpha_{2 n-k}, \alpha_{2 n-k+2}, \cdots, \alpha_{k-1}, \alpha_{0}}\right\|}
$$

Similar calculations as have been done in Lemma 4.8 will prove that

$$
\left\{e_{\alpha_{1}, \cdots, \alpha_{2 n-k}, \alpha_{2 n-k+2}, \cdots, \alpha_{k-1}, \alpha_{0}},:\left(\alpha_{1}, \cdots, \alpha_{2 n-k}, \alpha_{2 n-k+2}, \cdots, \alpha_{k-1}, \alpha_{0}\right) \in \mathbb{N}^{k-1}\right\}
$$

form an orthonormal basis for $\mathcal{H}$. By the same argument as used in the previous theorem, one proves that the representation satisfying $\pi\left(z_{2 n}\right)=\pi\left(z_{2 n-1}\right)=\cdots=\pi\left(z_{k+1}\right)=0$ and $\pi\left(z_{k}\right) u=t u$ is unique.

Case $2: k \leq n$.

First observe that the relations satisfied by $\pi\left(z_{1}\right), \pi\left(z_{2}\right), \cdots, \pi\left(z_{k}\right)$ are same as the defining relations of the odd dimensional quantum sphere $S_{q}^{2 k+1}$ for which we know that the claim holds. (Note that we can also proceed as in the previous case and establish the claim.)

We have shown so far that if there exists an irreducible representation $\pi$ such that $\pi\left(z_{2 n}\right)=$ $\pi\left(z_{2 n-1}\right)=\cdots=\pi\left(z_{k+1}\right)=0$, and $\pi\left(z_{k}\right) u=t u$ for $t \in T$, then it is unique. Existence of these representations still needs to be shown.

By Theorem 3.1, $C\left(S P_{q}(2 n) / S P_{q}(2 n-2)\right)$ is the $C^{*}$-subalgebra of $C\left(S P_{q}(2 n)\right)$ generated by $\left\{u_{m}^{2 n}\right\}_{m=1}^{2 n}$. Now, if we look at the relations $I_{s t}^{i j}$ involving $u_{m}^{2 n}$ and $u_{m}^{1}$ by putting $(i, j)=(1,1)$ and $(2 n, 1)$, we get the relations satisfied by generators of $C\left(H_{q}^{2 n}\right)$ where $z_{m}=u_{2 n+1-m}^{2 n}$. From the universal property of $C\left(H_{q}^{2 n}\right)$, there exists a map $\eta: C\left(H_{q}^{2 n}\right) \longrightarrow C\left(S P_{q}(2 n) / S P_{q}(2 n-2)\right)$ such that $\eta\left(z_{j}\right)=u_{2 n+1-j}^{2 n}$ for all $j \in\{1,2, \cdots, 2 n\}$.

Denote by $\omega_{k}$ the following word in the Weyl group of $s p_{2 n}$,

$$
\omega_{k}= \begin{cases}I & \text { if } k=1 \\ s_{1} s_{2} \cdots s_{k-1} & \text { if } 2 \leq k \leq n \\ s_{1} s_{2} \cdots s_{n-1} s_{n} s_{n-1} \cdots s_{2 n-k+1} & \text { if } n<k \leq 2 n\end{cases}
$$


Let $\eta_{t, \omega_{k}}=\pi_{t, \omega_{k}} \circ \eta$. Hence we have an irreducible representation $\eta_{t, \omega_{k}}$ of $C\left(H_{q}^{2 n}\right)$ such that $\eta_{t, \omega_{k}}\left(z_{2 n}\right)=\eta_{t, \omega_{k}}\left(z_{2 n-1}\right)=\cdots=\eta_{t, \omega_{k}}\left(z_{k+1}\right)=0$ and $\eta_{t, \omega_{k}}\left(z_{k}\right) u=t u$ where $1<k \leq 2 n$. This gives an explicit description of the irreducible representations satisfying these conditions.

For $k=1$, define $\eta_{t, I}: C\left(H_{q}^{2 n}\right) \rightarrow \mathbb{C}$ by $\eta_{t, I}\left(z_{j}\right)=t \delta_{1 j}$. The set $\left\{\eta_{t, I}: t \in T\right\}$ gives all one dimensional irreducible representations of $C\left(H_{q}^{2 n}\right)$. Also, it satisfies $\eta_{t, I}\left(z_{2 n}\right)=\eta_{t, I}\left(z_{2 n-1}\right)=$ $\cdots=\eta_{t, I}\left(z_{2}\right)=0$ and $\eta_{t, I}\left(z_{1}\right) u=t u$.

Corollary 4.12. The set $\left\{\eta_{t, \omega_{k}}: 1 \leq k \leq 2 n, t \in \mathbb{T}\right\}$ gives a complete list of irreducible representations of $C\left(H_{q}^{2 n}\right)$.

To get a faithful representation of $C\left(H_{q}^{2 n}\right)$, define $\eta_{\omega_{k}}: C\left(H_{q}^{2 n}\right) \rightarrow C(\mathbb{T}) \otimes \mathscr{T}^{\otimes k-1}$ by $\eta_{\omega_{k}}(a)(t)=\eta_{t, \omega_{k}}(a) \quad$ for all $a \in C\left(H_{q}^{2 n}\right)$.

Corollary 4.13. $\eta_{\omega_{2 n}}$ is a faithful representation of $C\left(H_{q}^{2 n}\right)$.

Proof: It is easy to see that any irreducible representation factors through $\eta_{\omega_{2 n}}$ as, $\omega_{k}$ is a subword of $\omega_{2 n}$. This proves the claim.

Theorem 4.14. The homomorphism $\eta: C\left(H_{q}^{2 n}\right) \rightarrow C\left(S P_{q}(2 n) / S P_{q}(2 n-2)\right)$ is an isomorphism.

Proof: Clearly $\eta$ is a surjective homomorphism as $\eta\left(z_{i}\right)$ are generators of $C\left(S P_{q}(2 n) / S P_{q}(2 n-\right.$ $2)$ ). It follows from Corollary 4.12 that all irreducible representations of $C\left(H_{q}^{2 n}\right)$ factor through $\eta$ which shows that $\eta$ is injective. This proves the claim.

We separate out some important facts that will be useful in determining the $K$-groups of $C\left(H_{q}^{2 n}\right)$.

Corollary 4.15. Let $C_{1}=C(\mathbb{T})$ and for $2 \leq k \leq 2 n$, let $C_{k}=\eta_{\omega_{k}}\left(C\left(H_{q}^{2 n}\right)\right)$. Then the set $\left\{\eta_{t, \omega_{l}}: 1 \leq l \leq k, t \in \mathbb{T}\right\}$ gives a complete list of irreducible representations of $C_{k}$.

Corollary 4.16. Let $\pi=\eta_{t, \omega_{k}}$. Then

1. for $1 \leq k \leq n$, one has

$$
\pi\left(z_{1}\right)^{\alpha_{1}} \cdots \pi\left(z_{k-1}\right)^{\alpha_{k-1}} 1_{\{1\}}\left(\pi\left(z_{k}^{*} z_{k}\right)\right)=C p_{\alpha_{1}, 0} \otimes p_{\alpha_{2}, 0} \otimes \ldots \otimes p_{\alpha_{k-1}, 0} .
$$

2. for $n<k \leq 2 n$, one has

$$
\begin{aligned}
& \pi\left(z_{1}\right)^{\alpha_{1}} \cdots \pi\left(z_{2 n-k}\right)^{\alpha_{2 n-k}} \pi\left(z_{k-1}\right)^{\alpha_{k-1}} \pi\left(z_{k-2}\right)^{\alpha_{k-2}} \cdots \pi\left(z_{2 n-k+2}\right)^{\alpha_{2 n-k+2}} \\
& {\left[\pi\left(z_{n}\right), \pi\left(z_{n+1}\right)\right]^{\alpha_{0}} 1_{\{1\}}\left(\pi\left(z_{k}^{*} z_{k}\right)\right)} \\
& =C p_{\alpha_{1}, 0} \otimes \cdots p_{\alpha_{2 n-k}, 0} \otimes p_{\alpha_{2 n-k+2}, 0} \otimes \cdots p_{\alpha_{n}, 0} \otimes p_{\alpha_{0}, 0} \otimes p_{\alpha_{n+1}, 0} \otimes \cdots \otimes p_{\alpha_{k-1}, 0},
\end{aligned}
$$

where $p_{i, j}$ is the rank one operator on $\ell^{2} 2(\mathbb{N})$ sending the basis element $e_{j}$ to $e_{i}$ and $C$ is some non-zero constant. 


\section{$5 \quad K$-groups of $C\left(H_{q}^{2 n}\right)$}

Neshveyev \& Tuset ([12]) proved the $K K$-equivalence of the $C^{*}$-algebra $C(G / K)$ and $C\left(G_{q} / K_{q}\right)$. Hence one can determine the $K$-groups of $C\left(G_{q} / K_{q}\right)$ from those of $C(G / K)$ via the equivalence. As a consequence, generators of the $K$-groups of $C\left(G_{q} / K_{q}\right)$ would be images of generators of the corresponding $K$-groups of $C(G / K)$ under the equivalence. However the proof of the equivalence that is known is existential in nature and it is hard to find any generator using that equivalence. Here we obtain the $K$-groups of $C\left(S P_{q}(2 n) / S P_{q}(2 n-2)\right)$ in a computationally more tractable way and give an explicit description of the generators of the $K$-groups.

We first derive certain exact sequences analogous to that for the odd dimensional quantum sphere (see [16]). We then apply the six-term sequence in $K$-theory to compute the $K$-groups of $C\left(S P_{q}(2 n) / S P_{q}(2 n-2)\right)$. Let $p_{i, j}$ be the rank one operator on $L_{2}(\mathbb{N})$ sending basis element $e_{j}$ to $e_{i}$ and $p$ be the operator $p_{0,0}$.

Lemma 5.1. Let $C_{1}=C(\mathbb{T})$ and for $2 \leq k \leq 2 n$, let $C_{k}=\eta_{\omega_{k}}\left(C\left(H_{q}^{2 n}\right)\right)$. Then $C(\mathbb{T}) \otimes$ $\mathcal{K}\left(\ell^{2}(\mathbb{N})\right)^{\otimes(k-1)}$ is contained in $C_{k}$. Moreover, for $2 \leq k \leq 2 n$ we have the exact sequence,

$$
0 \longrightarrow C(\mathbb{T}) \otimes \mathcal{K}\left(\ell^{2}(\mathbb{N})\right)^{\otimes(k-1)} \longrightarrow C_{k} \stackrel{\sigma_{k}}{\longrightarrow} C_{k-1} \longrightarrow 0 .
$$

where $\sigma_{k}$ is the restriction of $\left(1^{\otimes(k-1)} \otimes \sigma\right)$ to $C_{k}$ and $\sigma: \mathscr{T} \rightarrow \mathbb{C}$ is the homomorphism such that $\sigma(S)=1$.

Proof: First we prove that $C(\mathbb{T}) \otimes \mathcal{K}\left(\ell^{2}(\mathbb{N})\right)^{\otimes(k-1)}$ is contained in $C_{k}$. For $k \leq n$, it follows from a result of Sheu ([16], Theorem 4$)$ as $C_{k}$ is isomorphic to $C\left(S_{q}^{2 k-1}\right)$. For $k>n$, and $m \geq 0$,

$$
\eta_{\omega_{k}}\left(z_{k}^{m} 1_{\{1\}}\left(z_{k}^{*} z_{k}\right)\right)=t^{m} \otimes \underbrace{p \otimes p \otimes \ldots \otimes p}_{k-1}, \quad \eta_{\omega_{k}}\left(z_{k}^{* m} 1_{\{1\}}\left(z_{k}^{*} z_{k}\right)\right)=t^{-m} \otimes \underbrace{p \otimes p \otimes \ldots \otimes p}_{k-1} .
$$

Also from Corollary 4.16, it follows that

$$
\begin{aligned}
& \eta_{\omega_{k}}\left(\left(z_{1}\right)^{m_{1}} \cdots\left(z_{2 n-k}\right)^{m_{2 n-k}}\left(z_{k-1}\right)^{m_{k-1}}\left(z_{k-2}\right)^{m_{k-2}} \cdots\left(z_{2 n-k+2}\right)^{m_{2 n-k+2}}\right. \\
& \left.\left[\left(z_{n}\right),\left(z_{n+1}\right)\right]^{m_{0}} 1_{\{1\}}\left(z_{k}^{*} z_{k}\right)\right) \\
& =C t^{\left(\sum_{i=0, i \neq 2 n-k+1}^{k-1} m_{i}\right)+m_{0}} \\
& \quad p_{m_{1}, 0} \otimes \cdots p_{m_{2 n-k}, 0} \otimes p_{m_{2 n-k+2}} \otimes \cdots p_{m_{n}, 0} \otimes p_{m_{0}, 0} \otimes p_{m_{n+1}, 0} \otimes \cdots \otimes p_{m_{k-1}, 0} .
\end{aligned}
$$

which shows that $t \otimes p_{m_{1}, 0} \otimes p_{m_{2}, 0} \otimes \ldots \otimes p_{m_{k-1}, 0}$ and $1 \otimes p_{m_{1}, 0} \otimes p_{m_{2}, 0} \otimes \ldots \otimes p_{m_{k-1}, 0}$ are contained in $C_{k}$. Hence $C_{k}$ contains $C(\mathbb{T}) \otimes \mathcal{K}\left(\ell^{2}(\mathbb{N})\right)^{\otimes(k-1)}$.

It is easy to see that $\sigma_{k}$ vanishes on $C(\mathbb{T}) \otimes \mathcal{K}\left(\ell^{2}(\mathbb{N})\right)^{\otimes(k-1)}$. Also, it follows from Corollary 4.15 that any irreducible representation of $C_{k}$ is of the form $\eta_{t, \omega_{l}}$ where $l \leq k$ and $t \in \mathbb{T}$. Hence an irreducible representation of $C_{k}$ that vanishes on $C(\mathbb{T}) \otimes \mathcal{K}\left(\ell^{2}(\mathbb{N})\right)^{\otimes(k-1)}$ is of the form $\eta_{t, \omega_{l}}$ where $l \leq k-1$ and $t \in \mathbb{T}$. But this factors through $\sigma_{k}$. Thus we get the desired exact sequence. 
Remark 5.2. Neshveyev \& Tuset ([12]) obtained a composition series for $C\left(G_{q} / K_{q}\right)$ for any Poisson-Lie closed subgroup $K$ of $G$. In particular, when $K$ is $C\left(S P_{q}(2 n-2)\right)$, one gets a composition series for $C\left(S P_{q}(2 n) / S P_{q}(2 n-2)\right)$. Note that the series of exact sequence derived in the Lemma 5.1 is different from that given in [12].

Define, for $1 \leq k \leq 2 n$,

$$
u_{k}=t \otimes \underbrace{p \otimes p \otimes \ldots \otimes p}_{k-1}+1-1 \otimes \underbrace{p \otimes p \otimes \ldots \otimes p}_{k-1} .
$$

It is easy to check that $u_{k}=\eta_{\omega_{k}}\left(z_{k} 1_{\{1\}}\left(z_{k}^{*} z_{k}\right)+1-1_{\{1\}}\left(z_{k}^{*} z_{k}\right)\right)$. Therefore $u_{k}$ is an unitary operator which is contained in $C_{k}$.

Theorem 5.3. Let $1 \leq k \leq 2 n$. The $K$-groups $K_{0}\left(C_{k}\right)$ and $K_{1}\left(C_{k}\right)$ are both isomorphic to $\mathbb{Z}$ and in particular, $\left[u_{k}\right]$ form a $\mathbb{Z}$-basis for $K_{1}\left(C_{k}\right)$ and [1] form a $\mathbb{Z}$-basis for $K_{0}\left(C_{k}\right)$.

Proof: We apply induction on $k$. For $k=1$, this is clear. Assume the result is true for $k-1$. From Lemma 5.1, we have the short exact sequence

$$
0 \longrightarrow C(\mathbb{T}) \otimes \mathcal{K}\left(\ell^{2}(\mathbb{N})\right)^{\otimes(k-1)} \longrightarrow C_{k} \stackrel{\sigma_{k}}{\rightarrow} C_{k-1} \longrightarrow 0 .
$$

which gives rise to the following six-term sequence in $K$-theory.

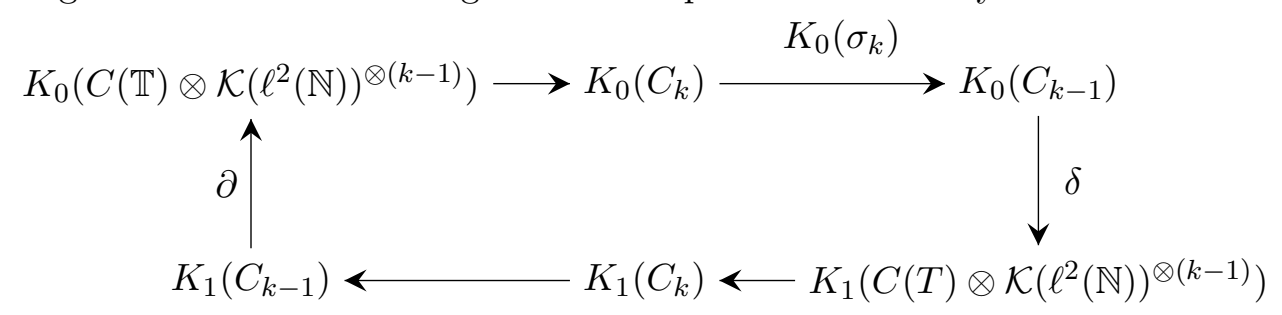

To compute the six term sequence, we determine $\delta$ and $\partial$. Since $\sigma_{k}(1)=1$, it follows that $\delta([1])=0$. Also, the operator $\widetilde{X}=t \otimes \underbrace{q^{N} \otimes q^{N} \otimes \ldots}_{k-2} \otimes S^{*}$ is in $C_{k}$ as $\eta_{\omega_{k}}\left(z_{k-1}\right)-\widetilde{X}$ lies in $C(\mathbb{T}) \otimes \mathcal{K}\left(\ell^{2}(\mathbb{N})\right)^{\otimes(k-1)}$. Let

$$
X=1_{\{1\}}\left(\widetilde{X}^{*} \tilde{X}\right) \tilde{X}+1-1_{\{1\}}\left(\tilde{X}^{*} \tilde{X}\right)
$$

Then $X$ is an isometry such that $\sigma_{k}(X)=u_{k-1}$ and hence

$$
\partial\left(\left[u_{k-1}\right]\right)=\left[1-X^{*} X\right]-\left[1-X X^{*}\right]=[1 \otimes \underbrace{p \otimes p \otimes \ldots \otimes p}_{k-1}] .
$$

Now by the Kunneth theorem for the tensor product of $C^{*}$-algebras (see [1] ), it follows that $C(\mathbb{T}) \otimes \mathcal{K}\left(\ell^{2}(\mathbb{N})\right)^{\otimes(k-1)}$ has $K_{0}$-group isomorphic to $\mathbb{Z}$ generated by $[1 \otimes \underbrace{p \otimes p \otimes \ldots \otimes p}_{k-1}]$ and $K_{1}$-group isomorphic to $\mathbb{Z}$ generated by $\left[u_{k}\right]$. Induction hypothesis and the above calculation 
shows that $\partial$ is an isomorphism and hence $K_{0}(i)$ is the zero map. Therefore $K_{0}\left(\sigma_{k}\right)$ is injective. Since $\delta$ is zero, $K_{0}\left(\sigma_{k}\right)$ is surjective. Hence $K_{0}\left(C_{k}\right)$ is isomorphic to $\mathbb{Z}$ and is generated by [1]. Similarly $K_{1}(i)$ is injective as $\delta$ is the zero map. Also, since $\partial$ is an isomorphism, $K_{1}\left(\sigma_{k}\right)$ is the zero map. This shows that $K_{1}(i)$ is surjective. Hence $K_{1}\left(C_{k}\right)$ is isomorphic to $\mathbb{Z}$ and is generated by $\left[u_{k}\right]$. This establishes the claim.

Acknowledgement: I would like to thank Prof. Arup Kumar Pal, my supervisor, for his constant support. I would also like to thank S. Sundar for useful discussions on various topics.

\section{References}

[1] Bruce Blackadar. K-theory for operator algebras, volume 5 of Mathematical Sciences Research Institute Publications. Cambridge University Press, Cambridge, second edition, 1998.

[2] Partha Sarathi Chakraborty and Arupkumar Pal. Characterization of spectral triples: A combinatorial approach. arXiv:math.OA/0305157, 2003.

[3] Partha Sarathi Chakraborty and Arupkumar Pal. Equivariant spectral triples on the quantum SU(2) group. K-Theory, 28(2):107-126, 2003.

[4] Partha Sarathi Chakraborty and Arupkumar Pal. Characterization of $\mathrm{SU}_{q}(\ell+1)$ equivariant spectral triples for the odd dimensional quantum spheres. J. Reine Angew. Math., 623:25-42, 2008.

[5] Partha Sarathi Chakraborty and S. Sundar. $K$-groups of the quantum homogeneous space $\mathrm{SU}_{q}(n) / \mathrm{SU}_{q}(n-2)$. Pacific J. Math., 252(2):275-292, 2011.

[6] Alain Connes. Cyclic cohomology, quantum group symmetries and the local index formula for $\mathrm{SU}_{q}(2)$. J. Inst. Math. Jussieu, 3(1):17-68, 2004.

[7] Ludwik Dąbrowski, Giovanni Landi, Andrzej Sitarz, Walter van Suijlekom, and Joseph C. Várilly. The Dirac operator on $\mathrm{SU}_{q}(2)$. Comm. Math. Phys., 259(3):729-759, 2005.

[8] William Fulton and Joe Harris. Representation theory, volume 129 of Graduate Texts in Mathematics. Springer-Verlag, New York, 1991. A first course, Readings in Mathematics.

[9] Jeong Hee Hong and Wojciech Szymański. Quantum spheres and projective spaces as graph algebras. Comm. Math. Phys., 232(1):157-188, 2002.

[10] Anatoli Klimyk and Konrad Schmüdgen. Quantum groups and their representations. Texts and Monographs in Physics. Springer-Verlag, Berlin, 1997. 
[11] Leonid I. Korogodski and Yan S. Soibelman. Algebras of functions on quantum groups. Part I, volume 56 of Mathematical Surveys and Monographs. American Mathematical Society, Providence, RI, 1998.

[12] Sergey Neshveyev and Lars Tuset. Quantized algebras of functions on homogeneous spaces with Poisson stabilizers. Comm. Math. Phys., 312(1):223-250, 2012.

[13] Arupkumar Pal and S. Sundar. Regularity and dimension spectrum of the equivariant spectral triple for the odd-dimensional quantum spheres. J. Noncommut. Geom., 4(3):389439, 2010.

[14] G. B. Podkolzin and L. I. Vainerman. Quantum Stiefel manifold and double cosets of quantum unitary group. Pacific J. Math., 188(1):179-199, 1999.

[15] Piotr Podleś. Symmetries of quantum spaces. Subgroups and quotient spaces of quantum SU(2) and SO(3) groups. Comm. Math. Phys., 170(1):1-20, 1995.

[16] Albert J. L. Sheu. Compact quantum groups and groupoid $C^{*}$-algebras. J. Funct. Anal., 144(2):371-393, 1997.

[17] L. L. Vaksman and Ya. S. Soibelman. An algebra of functions on the quantum group SU(2). Funktsional. Anal. i Prilozhen., 22(3):1-14, 96, 1988.

[18] L. L. Vaksman and Ya. S. Soibelman. Algebra of functions on the quantum group SU( $n+1)$, and odd-dimensional quantum spheres. Algebra i Analiz, 2(5):101-120, 1990.

[19] S. L. Woronowicz. Twisted SU(2) group. An example of a noncommutative differential calculus. Publ. Res. Inst. Math. Sci., 23(1):117-181, 1987.

[20] S. L. Woronowicz. Tannaka-Kren duality for compact matrix pseudogroups. Twisted $\mathrm{SU}(\mathrm{N})$ groups. Invent. Math. 93 (1988), no. 1, 35-76.

[21] D. P. Zhelobenko. Classical groups. Spectral analysis of finite-dimensional representations Uspehi Mat. Nauk, 1 (103):27-120, 17, 1962.

Bipul SaurabH (bipul9r@isid.ac.in)

Indian Statistical Institute, 7, SJSS Marg, New Delhi-110 016, INDIA 